\title{
TWO-PARAMETER QUANTUM GROUPS AND DRINFEL'D DOUBLES
}

\author{
GeOrgia BenkART ${ }^{1}$ \\ SARAH WITHERSPOON ${ }^{1}$
}

August 3, 2001

\begin{abstract}
We investigate two-parameter quantum groups corresponding to the general linear and special linear Lie algebras $\mathfrak{g l}_{n}$ and $\mathfrak{s l}_{n}$. We show that these quantum groups can be realized as Drinfel'd doubles of certain Hopf subalgebras with respect to Hopf pairings. Using the Hopf pairing, we construct a corresponding $R$-matrix and a quantum Casimir element. We discuss isomorphisms among these quantum groups and connections with multiparameter quantum groups.
\end{abstract}

\section{INTRODUCTION}

In this work we study two two-parameter quantum groups $\widetilde{U}=U_{r, s}\left(\mathfrak{g l}_{n}\right)$ and $U=U_{r, s}\left(\mathfrak{s l}_{n}\right)$ corresponding to the Lie algebras $\mathfrak{g l}_{n}$ and $\mathfrak{s l}_{n}$. Our Hopf algebra $\widetilde{U}$ is isomorphic as an algebra to Takeuchi's $U_{r, s^{-1}}$ (see [T]), but as a Hopf algebra, it has the opposite coproduct. (A different presentation of $U_{r, s^{-1}}$ was obtained by Kulish $[\mathrm{K}]$ (see also $[\mathrm{Ji}])$.) As an algebra, $\widetilde{U}$ has generators $e_{j}, f_{j},(1 \leq j<n)$, and $a_{i}^{ \pm 1}, b_{i}^{ \pm 1} \quad(1 \leq i \leq n)$, and defining relations given in (R1)-(R7) below. The elements $e_{j}, f_{j}, \omega_{j}^{ \pm 1},\left(\omega_{j}^{\prime}\right)^{ \pm 1}(1 \leq j<n)$, where $\omega_{j}=a_{j} b_{j+1}$ and $\omega_{j}^{\prime}=a_{j+1} b_{j}$, generate the subalgebra $U=U_{r, s}\left(\mathfrak{s l}_{n}\right)$.

We show that both $\widetilde{U}$ and $U$ may be realized as Drinfel'd doubles of certain Hopf subalgebras with respect to suitable Hopf pairings. Using the Hopf pairing, we construct an $R$-matrix for $\widetilde{U}$ (which also works for $U$ ). For $\widetilde{U}$-modules $M$ and $M^{\prime}$ in category $\mathcal{O}$ (defined in Section 4), there is an isomorphism $R_{M^{\prime}, M}: M^{\prime} \otimes M \rightarrow$ $M \otimes M^{\prime}$. Moreover, the $R$-matrix satisfies the quantum Yang-Baxter equation and the hexagon identities. In [BW2], the $R$-matrix will be used to establish an analogue of Schur-Weyl duality in this setting: $\widetilde{U}$ has a natural $n$-dimensional module $V$, and the centralizer algebra $\operatorname{End}_{\widetilde{U}}\left(V^{\otimes k}\right)$ is generated by a certain Hecke algebra $H_{k}(r, s)$. We construct a quantum Casimir element, which will play an essential role in [BW2] in proving that finite-dimensional modules in category $\mathcal{O}$ are completely reducible.

2000 Mathematics Subject Classification. Primary 17B37, 16W30, 16W35, 81R50.

${ }^{1}$ The authors gratefully acknowledge support from National Science Foundation Grant \#DMS-9970119, National Security Agency Grant \#MDA904-01-1-0067, and the hospitality of the Mathematical Sciences Research Institute, Berkeley.

Typeset by $\mathcal{A} \mathcal{M S}-\mathrm{TEX}_{\mathrm{E}}$ 
Jing's work [Ji], which treats the special case of $\mathfrak{g l}_{2}$, adopts exactly the opposite approach to the one of this paper - it derives an analogue of the algebra $\widetilde{U}$ from one particular solution $R$ of the quantum Yang-Baxter equation. Similarly, Chin and Musson $[\mathrm{ChM}]$ and Dobrev and Parashar $[\mathrm{DP}]$ study multiparameter quantum universal enveloping algebras defined as duals of quantum function algebras arising from $R$-matrices. In Section 6, we relate the two-parameter quantum groups considered here with certain special cases of these multiparameter quantum groups. Moreover, we determine conditions for isomorphisms among the two-parameter quantum groups. In particular, the standard one-parameter quantum group $U_{q}\left(\mathfrak{s l}_{2}\right)$ of [Ja] is isomorphic to a quotient of $U_{r, s}\left(\mathfrak{s l}_{2}\right)$ by the ideal generated by $\omega_{1}^{\prime}-\omega_{1}^{-1}$ whenever $q$ is a square root of $r s^{-1}$. However, for $n \geq 3$, no such isomorphism exists (see Proposition 6.1).

Our motivation to study these two-parameter quantum groups came from our work [BW1] on down-up algebras. Down-up algebras were introduced in $[\mathrm{BR}]$ as a generalization of the algebra generated by the down and up operators on posets. They are unital associative algebras $A(\alpha, \beta, \gamma)$ over a field $\mathbb{K}$ having generators $d, u$ which satisfy the defining relations

$$
\begin{gathered}
d^{2} u=\alpha d u d+\beta u d^{2}+\gamma d \\
d u^{2}=\alpha u d u+\beta u^{2} d+\gamma u
\end{gathered}
$$

where $\alpha, \beta, \gamma$ are fixed but arbitrary scalars in $\mathbb{K}$. If $\gamma \neq 0$, then the down-up algebra $A(\alpha, \beta, \gamma)$ is isomorphic to $A(\alpha, \beta, 1)$. Thus, there are basically two different cases: $\gamma=0$ and $\gamma=1$. Examples of down-up algebras include the universal enveloping algebras of $\mathfrak{s l}_{2}$, of the Heisenberg Lie algebra, and of the Lie superalgebra $\mathfrak{o s p}(1,2)$, which are $A(2,-1,1), A(2,-1,0)$, and $A(0,-1,1)$, respectively, and many of Witten's deformations of $U\left(\mathfrak{s l}_{2}\right)$ (see [B]). Down-up algebras exhibit many striking features including a Poincaré-Birkhoff-Witt type basis and a well-behaved representation theory ([BR], $[\mathrm{KMP}],[\mathrm{CaM}],[\mathrm{Jor}],[\mathrm{KK} 1],[\mathrm{KK} 2],[\mathrm{Ku}],[\mathrm{BL}, \mathrm{Sec}$. 4]). They are Noetherian domains whenever $\beta \neq 0$.

Essential to the structure of $A(\alpha, \beta, \gamma)$ are the roots of the equation

$$
0=t^{2}-\alpha t-\beta=(t-r)(t-s)
$$

Thus, $\alpha=r+s$ and $\beta=-r s$. When $r s \neq 0$ and $\gamma=0$, the down-up algebra $A(\alpha, \beta, 0)=A(r+s,-r s, 0)$ can be extended by automorphisms to give a Hopf algebra $B(r+s,-r s, 0)$ (see [BW1]). This Hopf algebra is isomorphic to a subalgebra of $U_{r, s}\left(\mathfrak{s l}_{3}\right)$ when $r$ and $s$ are not roots of unity and to a quotient of a subalgebra when they are. It seemed natural to expect that there is a Drinfel'd double (quantum double) of the subalgebra, which yields a quantum group that depends on the two parameters $r$ and $s$. In fact, that quantum group is $U_{r, s}\left(\mathfrak{s l}_{3}\right)$. That result is a very special case of our theorem showing that $U$ and $\widetilde{U}$ are Drinfel'd doubles.

Throughout we will be working over a field $\mathbb{K}$, which is required to be algebraically closed from Section 3 to the end of the paper. 


\section{§1. PReliminaries}

Assume $\Phi$ is a finite root system of type $\mathrm{A}_{n-1}$ with $\Pi$ a base of simple roots. We regard $\Phi$ as a subset of a Euclidean space $E=\mathbb{R}^{n}$ with an inner product $\langle$,$\rangle . We$ let $\epsilon_{1}, \ldots, \epsilon_{n}$ denote an orthonormal basis of $E$, and suppose $\Pi=\left\{\alpha_{j}=\epsilon_{j}-\epsilon_{j+1} \mid\right.$ $j=1, \ldots, n-1\}$ and $\Phi=\left\{\epsilon_{i}-\epsilon_{j} \mid 1 \leq i \neq j \leq n\right\}$.

Fix nonzero elements $r, s$ in a field $\mathbb{K}$. Here we assume $r \neq s$.

Let $\widetilde{U}=U_{r, s}\left(\mathfrak{g l}_{n}\right)$ be the unital associative algebra over $\mathbb{K}$ generated by elements $e_{j}, f_{j},(1 \leq j<n)$, and $a_{i}^{ \pm 1}, b_{i}^{ \pm 1} \quad(1 \leq i \leq n)$, which satisfy the following relations.

(R1) The $a_{i}^{ \pm 1}, b_{j}^{ \pm 1}$ all commute with one another and $a_{i} a_{i}^{-1}=b_{j} b_{j}^{-1}=1$,

(R2) $a_{i} e_{j}=r^{\left\langle\epsilon_{i}, \alpha_{j}\right\rangle} e_{j} a_{i}$ and $a_{i} f_{j}=r^{-\left\langle\epsilon_{i}, \alpha_{j}\right\rangle} f_{j} a_{i}$,

(R3) $b_{i} e_{j}=s^{\left\langle\epsilon_{i}, \alpha_{j}\right\rangle} e_{j} b_{i}$ and $b_{i} f_{j}=s^{-\left\langle\epsilon_{i}, \alpha_{j}\right\rangle} f_{j} b_{i}$,

(R4) $\left[e_{i}, f_{j}\right]=\frac{\delta_{i, j}}{r-s}\left(a_{i} b_{i+1}-a_{i+1} b_{i}\right)$,

(R5) $\left[e_{i}, e_{j}\right]=\left[f_{i}, f_{j}\right]=0$ if $|i-j|>1$,

(R6) $e_{i}^{2} e_{i+1}-(r+s) e_{i} e_{i+1} e_{i}+r s e_{i+1} e_{i}^{2}=0$, $e_{i} e_{i+1}^{2}-(r+s) e_{i+1} e_{i} e_{i+1}+r s e_{i+1}^{2} e_{i}=0$,

(R7) $f_{i}^{2} f_{i+1}-\left(r^{-1}+s^{-1}\right) f_{i} f_{i+1} f_{i}+r^{-1} s^{-1} f_{i+1} f_{i}^{2}=0$, $f_{i} f_{i+1}^{2}-\left(r^{-1}+s^{-1}\right) f_{i+1} f_{i} f_{i+1}+r^{-1} s^{-1} f_{i+1}^{2} f_{i}=0$.

The relations in (R6) are just the two defining relations of the down-up algebra $A(r+s,-r s, 0)$, while those in (R7) are the defining relations of $A\left(r^{-1}+\right.$ $\left.s^{-1},-r^{-1} s^{-1}, 0\right)$. In fact these two down-up algebras are isomorphic via the map that takes $d$ to $u^{\prime}$ and $u$ to $d^{\prime}$ (assuming $d^{\prime}, u^{\prime}$ are the generators of the latter) (see $[\mathrm{BR}])$.

We will be interested in the subalgebra $U=U_{r, s}\left(\mathfrak{s l}_{n}\right)$ of $\widetilde{U}=U_{r, s}\left(\mathfrak{g l}_{n}\right)$ generated by the elements $e_{j}, f_{j}, \omega_{j}$, and $\omega_{j}^{\prime}(1 \leq j<n)$, where

$$
\omega_{j}=a_{j} b_{j+1} \text { and } \omega_{j}^{\prime}=a_{j+1} b_{j} .
$$

These elements satisfy (R5)-(R7) along with the following relations:

$\left(\mathrm{R} 1^{\prime}\right)$ The $\omega_{i}^{ \pm 1}, \omega_{j}^{ \pm 1}$ all commute with one another and $\omega_{i} \omega_{i}^{-1}=\omega_{j}^{\prime}\left(\omega_{j}^{\prime}\right)^{-1}=1$,

$\left(\mathrm{R} 2^{\prime}\right) \omega_{i} e_{j}=r^{\left\langle\epsilon_{i}, \alpha_{j}\right\rangle} s^{\left\langle\epsilon_{i+1}, \alpha_{j}\right\rangle} e_{j} \omega_{i}$ and $\omega_{i} f_{j}=r^{-\left\langle\epsilon_{i}, \alpha_{j}\right\rangle} s^{-\left\langle\epsilon_{i+1}, \alpha_{j}\right\rangle} f_{j} \omega_{i}$,

(R3') $\omega_{i}^{\prime} e_{j}=r^{\left\langle\epsilon_{i+1}, \alpha_{j}\right\rangle} s^{\left\langle\epsilon_{i}, \alpha_{j}\right\rangle} e_{j} \omega_{i}^{\prime}$ and $\omega_{i}^{\prime} f_{j}=r^{-\left\langle\epsilon_{i+1}, \alpha_{j}\right\rangle} s^{-\left\langle\epsilon_{i}, \alpha_{j}\right\rangle} f_{j} \omega_{i}^{\prime}$,

$\left(\mathrm{R} 4^{\prime}\right)\left[e_{i}, f_{j}\right]=\frac{\delta_{i, j}}{r-s}\left(\omega_{i}-\omega_{i}^{\prime}\right)$. 
When $r=q$ and $s=q^{-1}$, the algebra $U_{r, s}\left(\mathfrak{g l}_{n}\right)$ modulo the ideal generated by the elements $b_{i}-a_{i}^{-1}, 1 \leq i \leq n$, is just the quantum general linear group $U_{q}\left(\mathfrak{g l}_{n}\right)$, and $U_{r, s}\left(\mathfrak{s l}_{n}\right)$ modulo the ideal generated by the elements $\omega_{j}^{\prime}-\omega_{j}^{-1}, 1 \leq j<n$, is $U_{q}\left(\mathfrak{s l}_{n}\right)$.

Let $Q=\mathbb{Z} \Phi$ denote the root lattice and set $Q^{+}=\sum_{i=1}^{n-1} \mathbb{Z}_{\geq 0} \alpha_{i}$. Then for any $\zeta=\sum_{i=1}^{n-1} \zeta_{i} \alpha_{i} \in Q$, we adopt the shorthand

$$
\omega_{\zeta}=\omega_{1}^{\zeta_{1}} \cdots \omega_{n-1}^{\zeta_{n-1}}, \quad \omega_{\zeta}^{\prime}=\left(\omega_{1}^{\prime}\right)^{\zeta_{1}} \cdots\left(\omega_{n-1}^{\prime}\right)^{\zeta_{n-1}}
$$

The following lemma is straightforward to check.

Lemma 1.3. Suppose that $\zeta=\sum_{i=1}^{n-1} \zeta_{i} \alpha_{i} \in Q$. Then

$$
\begin{array}{ll}
w_{\zeta} e_{i}=r^{-\left\langle\epsilon_{i+1}, \zeta\right\rangle} s^{-\left\langle\epsilon_{i}, \zeta\right\rangle} e_{i} w_{\zeta} & w_{\zeta} f_{i}=r^{\left\langle\epsilon_{i+1}, \zeta\right\rangle} s^{\left\langle\epsilon_{i}, \zeta\right\rangle} f_{i} w_{\zeta} \\
w_{\zeta}^{\prime} e_{i}=r^{-\left\langle\epsilon_{i}, \zeta\right\rangle} s^{-\left\langle\epsilon_{i+1}, \zeta\right\rangle} e_{i} w_{\zeta}^{\prime} & w_{\zeta}^{\prime} f_{i}=r^{\left\langle\epsilon_{i}, \zeta\right\rangle} s^{\left\langle\epsilon_{i+1}, \zeta\right\rangle} f_{i} w_{\zeta}^{\prime} .
\end{array}
$$

The algebras $\widetilde{U}$ and $U$ are Hopf algebras, where the $a_{i}^{ \pm 1}, b_{i}^{ \pm 1}$ are group-like elements, and the remaining coproducts are determined by

$$
\Delta\left(e_{i}\right)=e_{i} \otimes 1+\omega_{i} \otimes e_{i}, \quad \Delta\left(f_{i}\right)=1 \otimes f_{i}+f_{i} \otimes \omega_{i}^{\prime}
$$

This forces the counit and antipode maps to be

$$
\begin{aligned}
& \varepsilon\left(a_{i}\right)=\varepsilon\left(b_{i}\right)=1, \quad S\left(a_{i}\right)=a_{i}^{-1}, \quad S\left(b_{i}\right)=b_{i}^{-1} \\
& \varepsilon\left(e_{i}\right)=\varepsilon\left(f_{i}\right)=0, \quad S\left(e_{i}\right)=-\omega_{i}^{-1} e_{i}, \quad S\left(f_{i}\right)=-f_{i}\left(\omega_{i}^{\prime}\right)^{-1} .
\end{aligned}
$$

\section{§2. DRINFEL'D DOUBLES}

A Hopf pairing of two Hopf algebras $H$ and $H^{\prime}$ is a bilinear form on $H^{\prime} \times H$ satisfying the following properties (see [Jo, 3.2.1]):

$$
\begin{aligned}
& \text { 1)(i) }(1, h)=\varepsilon_{H}(h), \quad\left(h^{\prime}, 1\right)=\varepsilon_{H^{\prime}}\left(h^{\prime}\right) \\
& \text { (ii) }\left(h^{\prime}, h k\right)=\left(\Delta_{H^{\prime}}\left(h^{\prime}\right), h \otimes k\right)=\sum\left(h_{(1)}^{\prime}, h\right)\left(h_{(2)}^{\prime}, k\right) \\
& \text { (iii) }\left(h^{\prime} k^{\prime}, h\right)=\left(h^{\prime} \otimes k^{\prime}, \Delta_{H}(h)\right)=\sum\left(h^{\prime}, h_{(1)}\right)\left(k^{\prime}, h_{(2)}\right)
\end{aligned}
$$

for all $h, k \in H$ and $h^{\prime}, k^{\prime} \in H^{\prime}$, where $\varepsilon_{H}$ and $\varepsilon_{H^{\prime}}$ denote the counits of $H$ and $H^{\prime}$, respectively, and $\Delta_{H}$ and $\Delta_{H^{\prime}}$ are their coproducts. It is a consequence of the defining properties that a Hopf pairing satisfies

$$
\left(S_{H^{\prime}}\left(h^{\prime}\right), h\right)=\left(h^{\prime}, S_{H}(h)\right)
$$


for all $h \in H$ and $h^{\prime} \in H^{\prime}$, where $S_{H}$ and $S_{H^{\prime}}$ denote the respective antipodes of $H$ and $H^{\prime}$.

Assume $\widetilde{B}$ is the Hopf subalgebra of $\widetilde{U}$ generated by $e_{j}, \omega_{j}^{ \pm 1}(1 \leq j<n)$, and $a_{n}^{ \pm 1}$. Let $\left(\widetilde{B}^{\prime}\right)^{\text {coop }}$ be the Hopf algebra having the opposite coproduct to the Hopf subalgebra of $\widetilde{U}$ generated by $f_{j},\left(\omega_{j}^{\prime}\right)^{ \pm 1}(1 \leq j<n)$, and $b_{n}^{ \pm 1}$. Similarly $B$ is the Hopf subalgebra of $U$ generated by $e_{j}, \omega_{j}^{ \pm 1}(1 \leq j<n)$, and $\left(B^{\prime}\right)^{\text {coop }}$ is generated by $f_{j},\left(\omega_{j}^{\prime}\right)^{ \pm 1}(1 \leq j<n)$.

Lemma 2.2. There are Hopf pairings of $\widetilde{B}$ and $\widetilde{B}^{\prime}$, respectively of $B$ and $B^{\prime}$.

Proof. We begin by defining a bilinear form for $\widetilde{B}^{\prime} \times \widetilde{B}$ first on the generators:

$$
\begin{aligned}
\left(f_{i}, e_{j}\right) & =\frac{\delta_{i, j}}{s-r} \\
\left(\omega_{i}^{\prime}, \omega_{j}\right) & =r^{\left\langle\epsilon_{j}, \alpha_{i}\right\rangle} s^{\left\langle\epsilon_{j+1}, \alpha_{i}\right\rangle}=r^{-\left\langle\epsilon_{i+1}, \alpha_{j}\right\rangle} s^{-\left\langle\epsilon_{i}, \alpha_{j}\right\rangle} \\
\left(b_{n}, a_{n}\right) & =1, \quad\left(b_{n}, \omega_{j}\right)=s^{-\left\langle\epsilon_{n}, \alpha_{j}\right\rangle}, \quad\left(\omega_{i}^{\prime}, a_{n}\right)=r^{\left\langle\epsilon_{n}, \alpha_{i}\right\rangle}
\end{aligned}
$$

for $(1 \leq i, j<n)$. If $\omega_{i}^{\prime}$ is replaced by $\left(\omega_{i}^{\prime}\right)^{-1}$ in the second or third line of $(2.3)$, we replace the image under the bilinear form by its inverse, and similarly for $\omega_{j}$ and $\omega_{j}^{-1}, a_{n}$ and $a_{n}^{-1}, b_{n}$ and $b_{n}^{-1}$. On all other pairs of generators the form is 0 .

In the second line of (2.3) we have applied the identity

$$
\left\langle\epsilon_{j}, \alpha_{i}\right\rangle=-\left\langle\epsilon_{i+1}, \alpha_{j}\right\rangle,
$$

which is quite useful in subsequent calculations.

The pairings in (2.3) may be extended to a bilinear form on $\widetilde{B}^{\prime} \times \widetilde{B}$ by requiring that (2.1)(i)-(iii) hold. We need only verify that the relations in $\widetilde{B}$ and $\widetilde{B}^{\prime}$ are preserved, ensuring that the bilinear form is well-defined. It will then be a Hopf pairing by definition. Restricting the form to $B^{\prime} \times B$ gives the desired Hopf pairing of $B$ and $B^{\prime}$.

It is straightforward to check that the bilinear form preserves all the relations among the $\omega_{i}^{ \pm 1}, a_{n}^{ \pm 1}$ in $\widetilde{B}$ and the $\left(\omega_{j}^{\prime}\right)^{ \pm 1}, b_{n}^{ \pm 1}$ in $\widetilde{B}^{\prime}$. We will verify that the form on $\widetilde{B}^{\prime} \times \widetilde{B}$ preserves one of the remaining relations in $\widetilde{B}$, and leave the other verifications to the reader. For each $i, 1 \leq i<n$, consider

$$
\left(X, e_{i}^{2} e_{i+1}-(r+s) e_{i} e_{i+1} e_{i}+r s e_{i+1} e_{i}^{2}\right),
$$

where $X$ is any word in the generators of $\widetilde{B}^{\prime}$. By definition, this is equal to

$$
\left(\Delta^{2}(X), e_{i} \otimes e_{i} \otimes e_{i+1}-(r+s) e_{i} \otimes e_{i+1} \otimes e_{i}+r s e_{i+1} \otimes e_{i} \otimes e_{i}\right) .
$$

In order for any one of these terms to be nonzero, $X$ must involve exactly two $f_{i}$ factors, one $f_{i+1}$ factor, and arbitrarily many $\left(\omega_{j}^{\prime}\right)^{ \pm 1}$ and $b_{n}^{ \pm 1}$ factors $(1 \leq j<n)$. First assume that $X=f_{i}^{2} f_{i+1}$. Then $\Delta^{2}(X)$ is equal to

$$
\left(\omega_{i}^{\prime} \otimes \omega_{i}^{\prime} \otimes f_{i}+\omega_{i}^{\prime} \otimes f_{i} \otimes 1+f_{i} \otimes 1 \otimes 1\right)^{2}\left(\omega_{i+1}^{\prime} \otimes \omega_{i+1}^{\prime} \otimes f_{i+1}+\omega_{i+1}^{\prime} \otimes f_{i+1} \otimes 1+f_{i+1} \otimes 1 \otimes 1\right) .
$$


The relevant terms of $\Delta^{2}(X)$ are

$$
\begin{gathered}
f_{i} \omega_{i}^{\prime} \omega_{i+1}^{\prime} \otimes f_{i} \omega_{i+1}^{\prime} \otimes f_{i+1}+\omega_{i}^{\prime} f_{i} \omega_{i+1}^{\prime} \otimes f_{i} \omega_{i+1}^{\prime} \otimes f_{i+1} \\
+f_{i} \omega_{i}^{\prime} \omega_{i+1}^{\prime} \otimes \omega_{i}^{\prime} f_{i+1} \otimes f_{i}+\omega_{i}^{\prime} f_{i} \omega_{i+1}^{\prime} \otimes \omega_{i}^{\prime} f_{i+1} \otimes f_{i} \\
\quad+\left(\omega_{i}^{\prime}\right)^{2} f_{i+1} \otimes f_{i} \omega_{i}^{\prime} \otimes f_{i}+\left(\omega_{i}^{\prime}\right)^{2} f_{i+1} \otimes \omega_{i}^{\prime} f_{i} \otimes f_{i}
\end{gathered}
$$

Therefore (2.5) becomes

$$
\begin{aligned}
& \left(f_{i} \omega_{i}^{\prime} \omega_{i+1}^{\prime}, e_{i}\right)\left(f_{i} \omega_{i+1}^{\prime}, e_{i}\right)\left(f_{i+1}, e_{i+1}\right)+\left(\omega_{i}^{\prime} f_{i} \omega_{i+1}^{\prime}, e_{i}\right)\left(f_{i} \omega_{i+1}^{\prime}, e_{i}\right)\left(f_{i+1}, e_{i+1}\right) \\
& -(r+s)\left(f_{i} \omega_{i}^{\prime} \omega_{i+1}^{\prime}, e_{i}\right)\left(\omega_{i}^{\prime} f_{i+1}, e_{i+1}\right)\left(f_{i}, e_{i}\right)-(r+s)\left(\omega_{i}^{\prime} f_{i} \omega_{i+1}^{\prime}, e_{i}\right)\left(\omega_{i}^{\prime} f_{i+1}, e_{i+1}\right)\left(f_{i}, e_{i}\right) \\
& \quad+r s\left(\left(\omega_{i}^{\prime}\right)^{2} f_{i+1}, e_{i+1}\right)\left(f_{i} \omega_{i}^{\prime}, e_{i}\right)\left(f_{i}, e_{i}\right)+r s\left(\left(\omega_{i}^{\prime}\right)^{2} f_{i+1}, e_{i+1}\right)\left(\omega_{i}^{\prime} f_{i}, e_{i}\right)\left(f_{i}, e_{i}\right) \\
& =\frac{1}{(s-r)^{3}}\left(1+\left(\omega_{i}^{\prime}, \omega_{i}\right)-(r+s)\left(\omega_{i}^{\prime}, \omega_{i+1}\right)-(r+s)\left(\omega_{i}^{\prime}, \omega_{i}\right)\left(\omega_{i}^{\prime}, \omega_{i+1}\right)\right. \\
& \left.\quad \quad+r s\left(\omega_{i}^{\prime}, \omega_{i+1}\right)^{2}+r s\left(\omega_{i}^{\prime}, \omega_{i+1}\right)^{2}\left(\omega_{i}^{\prime}, \omega_{i}\right)\right) \\
& =\frac{1}{(s-r)^{3}}\left(1+r s^{-1}-(r+s) r^{-1}-(r+s) s^{-1}+r^{-1} s+1\right)=0 .
\end{aligned}
$$

If $X=f_{i} f_{i+1} f_{i}$ or $X=f_{i+1} f_{i}^{2}$, then similar calculations show that (2.5) is equal to 0 . Finally if $X$ is any word involving exactly two $f_{i}$ factors, one $f_{i+1}$ factor, and arbitrarily many factors of $\left(\omega_{j}^{\prime}\right)^{ \pm 1}(1 \leq j<n)$ and $b_{n}^{ \pm 1}$, then $(2.5)$ will just be a scalar multiple of one of the quantities we have already calculated, and therefore will equal 0. (For example, if $X=f_{i}^{2} \omega_{j}^{\prime} f_{i+1}$, then (2.5) will be $\left(\omega_{j}^{\prime}, \omega_{i+1}\right)$ times the corresponding quantity for $X=f_{i}^{2} f_{i+1}$.)

Analogous calculations show that the relations in $\widetilde{B}^{\prime}$ are preserved.

As there is a Hopf pairing between $\widetilde{B}$ and $\widetilde{B}^{\prime}$, there is a skew-Hopf pairing between $\widetilde{B}$ and $\left(\widetilde{B}^{\prime}\right)^{\text {coop }}$, where the latter is $\widetilde{B}^{\prime}$ as an algebra, but with the opposite coproduct. Therefore, we may form the Drinfel'd double $D\left(\widetilde{B},\left(\widetilde{B}^{\prime}\right)^{\text {coop }}\right)$ as in [Jo, 3.2]. This is a Hopf algebra whose underlying coalgebra is $\widetilde{B} \otimes\left(\widetilde{B}^{\prime}\right)^{\text {coop }}$ (that is, $\widetilde{B} \otimes\left(\widetilde{B}^{\prime}\right)^{\text {coop }}$ as a vector space with the tensor product coalgebra structure). The algebra structure is given as follows: $\widetilde{B}$ and $\widetilde{B}^{\prime}$ are identified as algebras with $\widetilde{B} \otimes 1$ and $1 \otimes \widetilde{B}^{\prime}$ respectively in $D\left(\widetilde{B},\left(\widetilde{B}^{\prime}\right)^{\text {coop }}\right)$. Letting $a \in \widetilde{B}$ and $b \in \widetilde{B}^{\prime}$, we have $(a \otimes 1)(1 \otimes b)=a \otimes b$ and

$$
(1 \otimes b)(a \otimes 1)=\sum\left(S^{\mathrm{coop}}\left(b_{(1)}\right), a_{(1)}\right)\left(b_{(3)}, a_{(3)}\right) a_{(2)} \otimes b_{(2)},
$$

where $S^{\text {coop }}$ denotes the antipode for $\left(\widetilde{B}^{\prime}\right)^{\text {coop }}$. (This expression looks different from [Jo, Lemma 3.2.2 (iii)] as we have written our bilinear form in the reverse order.) A similar construction applies to $B$ and $B^{\prime}$.

Theorem 2.7. $D\left(\widetilde{B},\left(\widetilde{B}^{\prime}\right)^{\mathrm{coop}}\right)$ is isomorphic to $\widetilde{U}$, and $D\left(B,\left(B^{\prime}\right)^{\mathrm{coop}}\right)$ is isomorphic to $U$.

Proof. We will prove the first statement, and the second will follow by restricting to fewer generators. We will denote the image $e_{i} \otimes 1$ of $e_{i}$ in $D\left(\widetilde{B},\left(\widetilde{B}^{\prime}\right)^{\text {coop }}\right)$ by $\check{e}_{i}$, 
and similarly for $\omega_{i}, a_{n}, f_{i}, \omega_{i}^{\prime}, b_{n}$. Define a map $\varphi: D\left(\widetilde{B},\left(\widetilde{B}^{\prime}\right)^{\text {coop }}\right) \rightarrow \widetilde{U}$ by

$$
\begin{aligned}
\varphi\left(\check{e}_{i}\right) & =e_{i}, \quad \varphi\left(\check{f}_{i}\right)=f_{i}, \\
\varphi\left(\check{\omega}_{i}^{ \pm 1}\right)=\omega_{i}^{ \pm 1}, \quad \varphi\left(\left(\check{\omega}_{i}^{\prime}\right)^{ \pm 1}\right) & =\left(\omega_{i}^{\prime}\right)^{ \pm 1}, \quad \varphi\left(\check{a}_{n}^{ \pm 1}\right)=a_{n}^{ \pm 1}, \quad \varphi\left(\check{b}_{n}^{ \pm 1}\right)=b_{n}^{ \pm 1} .
\end{aligned}
$$

First notice that by definition, $\varphi$ preserves the coalgebra structures, the relations in $\widetilde{B}$, and the relations in $\widetilde{B}^{\prime}$. Next we will verify that the mixed relations in $D\left(\widetilde{B},\left(\widetilde{B}^{\prime}\right)^{\text {coop }}\right)$ correspond to those in $\widetilde{U}$.

To calculate $\check{f}_{j} \check{e}_{i}$, we use

$$
\begin{aligned}
& \Delta^{2}\left(e_{i}\right)=e_{i} \otimes 1 \otimes 1+\omega_{i} \otimes e_{i} \otimes 1+\omega_{i} \otimes \omega_{i} \otimes e_{i}, \\
& \left(\Delta^{\mathrm{coop}}\right)^{2}\left(f_{j}\right)=1 \otimes 1 \otimes f_{j}+1 \otimes f_{j} \otimes \omega_{j}^{\prime}+f_{j} \otimes \omega_{j}^{\prime} \otimes \omega_{j}^{\prime}, \\
& \text { and } \quad S^{\operatorname{coop}}\left(f_{j}\right)=-f_{j}\left(\omega_{j}^{\prime}\right)^{-1},
\end{aligned}
$$

so that

$$
\begin{aligned}
\check{f}_{j} \check{e}_{i} & =\left(-f_{j}\left(\omega_{j}^{\prime}\right)^{-1}, e_{i}\right)\left(\omega_{j}^{\prime}, 1\right) \check{\omega}_{j}^{\prime}+\left(1, \omega_{i}\right)\left(\omega_{j}^{\prime}, 1\right) \check{e}_{i} \check{f}_{j}+\left(1, \omega_{i}\right)\left(f_{j}, e_{i}\right) \check{\omega}_{i} \\
& =-\frac{\delta_{i, j}}{s-r} \check{\omega}_{j}^{\prime}+\check{e}_{i} \check{f}_{j}+\frac{\delta_{i, j}}{s-r} \check{\omega}_{i} .
\end{aligned}
$$

That is, $\left[\check{e}_{i}, \check{f}_{j}\right]=\delta_{i, j}(s-r)^{-1}\left(\check{\omega}_{i}-\check{\omega}_{i}^{\prime}\right)$. Applying $\varphi$ gives the desired relation in $\widetilde{U}$.

We leave verification of the remaining relations to the reader. As $\widetilde{U}$ is generated by $e_{i}, f_{i}, \omega_{i}^{ \pm 1},\left(\omega_{i}^{\prime}\right)^{ \pm 1}(1 \leq i<n), a_{n}$ and $b_{n}$, the map $\varphi$ is surjective, and there is an obvious inverse map.

\section{§3. Weight MOdules}

Let $\Lambda=\mathbb{Z} \epsilon_{1} \oplus \cdots \oplus \mathbb{Z} \epsilon_{n}$, which is the weight lattice of $\mathfrak{g l}_{n}$. Corresponding to any $\lambda \in \Lambda$ is an algebra homomorphism $\hat{\lambda}$ from the subalgebra $\widetilde{U}^{0}$ of $\widetilde{U}$ generated by the elements $a_{i}^{ \pm 1}, b_{i}^{ \pm 1}(1 \leq i \leq n)$ to $\mathbb{K}$ given by

$$
\hat{\lambda}\left(a_{i}\right)=r^{\left\langle\epsilon_{i}, \lambda\right\rangle} \quad \text { and } \quad \hat{\lambda}\left(b_{i}\right)=s^{\left\langle\epsilon_{i}, \lambda\right\rangle} .
$$

The restriction $\hat{\lambda}: U^{0} \rightarrow \mathbb{K}$ to the subalgebra $U^{0}$ of $U$ generated by $\omega_{j}^{ \pm 1},\left(\omega_{j}^{\prime}\right)^{ \pm 1}$ $(1 \leq j<n)$ satisfies

$$
\hat{\lambda}\left(\omega_{j}\right)=r^{\left\langle\epsilon_{j}, \lambda\right\rangle} s^{\left\langle\epsilon_{j+1}, \lambda\right\rangle} \quad \text { and } \quad \hat{\lambda}\left(\omega_{j}^{\prime}\right)=r^{\left\langle\epsilon_{j+1}, \lambda\right\rangle} s^{\left\langle\epsilon_{j}, \lambda\right\rangle}
$$

Let $M$ be a module for $\widetilde{U}=U_{r, s}\left(\mathfrak{g l}_{n}\right)$ of dimension $d<\infty$. If $\mathbb{K}$ is algebraically closed (which will be our assumption throughout the remainder of this work), then

$$
M=\bigoplus_{\chi} M_{\chi}
$$


where each $\chi: \widetilde{U}^{0} \rightarrow \mathbb{K}$ is an algebra homomorphism, and $M_{\chi}$ is the generalized eigenspace given by

$$
M_{\chi}=\left\{m \in M \mid\left(a_{i}-\chi\left(a_{i}\right) 1\right)^{d} m=0=\left(b_{i}-\chi\left(b_{i}\right) 1\right)^{d} m, \text { for all } i\right\} .
$$

When $M_{\chi} \neq 0$ we say that $\chi$ is a weight and $M_{\chi}$ is the corresponding weight space. (If $M$ decomposes into genuine eigenspaces relative to $\widetilde{U}^{0}$ (resp. $U^{0}$ ), then we say that $\widetilde{U}^{0}$ (resp. $U^{0}$ ) acts semisimply on $M$.)

From relations (R2) and (R3) we deduce that

$$
\begin{aligned}
& e_{j} M_{\chi} \subseteq M_{\chi \cdot \widehat{\alpha_{j}}} \\
& f_{j} M_{\chi} \subseteq M_{\chi \cdot\left(\widehat{-\alpha}_{j}\right)}
\end{aligned}
$$

where $\widehat{\alpha_{j}}$ is as in (3.1), and $\chi \cdot \psi$ is the homomorphism with values $(\chi \cdot \psi)\left(a_{i}\right)=$ $\chi\left(a_{i}\right) \psi\left(a_{i}\right)$ and $(\chi \cdot \psi)\left(b_{i}\right)=\chi\left(b_{i}\right) \psi\left(b_{i}\right)$. In fact, if $\left(a_{i}-\chi\left(a_{i}\right) 1\right)^{k} m=0$, then $\left(a_{i}-\chi\left(a_{i}\right) r^{\left\langle\epsilon_{i}, \alpha_{j}\right\rangle} 1\right)^{k} e_{j} m=0$, and similarly for $b_{i}$ and for $f_{j}$. This can be used to show that the sum of the eigenspaces is a submodule of $M$, and so if $M$ is simple, this sum must be $M$ itself. Thus, in (3.3), we may replace the power $d$ by 1 whenever $M$ is simple, and $\widetilde{U}^{0}$ must act semisimply in this case. We also can see from (3.4) that for each simple $M$ there is a homomorphism $\chi$ so that all the weights of $M$ are of the form $\chi \cdot \hat{\zeta}$, where $\zeta \in Q$.

When all the weights of a module $M$ are of the form $\hat{\lambda}$, where $\lambda \in \Lambda$, then for brevity we say that $M$ has weights in $\Lambda$. Rather than writing $M_{\hat{\lambda}}$ for the weight space, we simplify the notation by writing $M_{\lambda}$. Note then (3.4) can be rewritten as $e_{j} M_{\lambda} \subseteq M_{\lambda+\alpha_{j}}$ and $f_{j} M_{\lambda} \subseteq M_{\lambda-\alpha_{j}}$. Any simple $\widetilde{U}$-module having one weight in $\Lambda$ has all its weights in $\Lambda$.

We would like to argue that when $r s^{-1}$ is not a root of unity, the elements $e_{j}$ and $f_{j}$ act nilpotently on any finite-dimensional module. For this we require the following result.

Proposition 3.5. Suppose $\hat{\zeta}=\hat{\eta}$, where $\zeta, \eta \in Q$. If $\mathrm{rs}^{-1}$ is not a root of unity, then $\zeta=\eta$.

Proof. We will prove that when $\hat{\zeta}=\hat{\eta}$ as homomorphisms on the subalgebra $U^{0}$ generated by the $\omega_{i}, \omega_{i}^{\prime}$, then $\zeta=\eta$, so the result holds for $U$ as well as for $\widetilde{U}$. We may assume $\zeta=\sum_{i=1}^{n-1} \zeta_{i} \alpha_{i}$ and $\eta=\sum_{i=1}^{n-1} \eta_{i} \alpha_{i}$. The condition that $\hat{\zeta}=\hat{\eta}$ gives the equations

$$
\begin{aligned}
\hat{\zeta}\left(\omega_{i}\right) & =r^{\left\langle\epsilon_{i}, \zeta\right\rangle} s^{\left\langle\epsilon_{i+1}, \zeta\right\rangle}=r^{\zeta_{i}-\zeta_{i-1}} s^{\zeta_{i+1}-\zeta_{i}} \\
& =\hat{\eta}\left(\omega_{i}\right)=r^{\eta_{i}-\eta_{i-1}} s^{\eta_{i+1}-\eta_{i}} \\
\hat{\zeta}\left(\omega_{i}^{\prime}\right) & =r^{\left\langle\epsilon_{i+1}, \zeta\right\rangle} s^{\left\langle\epsilon_{i}, \zeta\right\rangle}=r^{\zeta_{i+1}-\zeta_{i}} s^{\zeta_{i}-\zeta_{i-1}} \\
& =\hat{\eta}\left(\omega_{i}^{\prime}\right)=r^{\eta_{i+1}-\eta_{i}} s^{\eta_{i}-\eta_{i-1}}
\end{aligned}
$$


where $\zeta_{0}=\zeta_{n}=0=\eta_{0}=\eta_{n}$. Letting $\mu_{i}=\zeta_{i}-\eta_{i}$, we may rewrite the above equations as

$$
\begin{aligned}
& r^{\mu_{i}-\mu_{i-1}} s^{\mu_{i+1}-\mu_{i}}=1 \\
& r^{\mu_{i+1}-\mu_{i}} s^{\mu_{i}-\mu_{i-1}}=1 .
\end{aligned}
$$

Combining these we have

$$
\begin{aligned}
& r^{\mu_{i+2}-\mu_{i+1}-\mu_{i}+\mu_{i-1}}=1 \\
& s^{\mu_{i+2}-\mu_{i+1}-\mu_{i}+\mu_{i-1}}=1
\end{aligned}
$$

for $i=1, \ldots, n-2$. Since we are assuming that $r s^{-1}$ is not a root of unity, not both $r$ and $s$ are roots of unity, so from these relations we see that

$$
\mu_{i+2}-\mu_{i+1}-\mu_{i}+\mu_{i-1}=0
$$

for $i=1, \ldots, n-2$. We claim that the solution to the system of equations given by (3.10) satisfies

$$
\mu_{2 k}=k \mu_{2} \quad \text { and } \quad \mu_{2 k+1}=k \mu_{2}+\mu_{1} .
$$

This is true for $\mu_{3}$ as $\mu_{0}=0$. Moreover, $\mu_{4}=\mu_{3}+\mu_{2}-\mu_{1}=2 \mu_{2}$. An easy induction proves the rest. Now $\mu_{n}=0$, and using that fact in (3.10) we have

$$
\mu_{n-1}=-\mu_{n-2}+\mu_{n-3} .
$$

If $n=2 m$ for some $m$, then (3.11) and (3.12) give $\mu_{2}=0$. From (3.6), we have $\left(r s^{-1}\right)^{\mu_{1}}=1$, and because $r s^{-1}$ is not a root of unity, this says $\mu_{1}=0$. The relations in (3.11) then show $\mu_{i}=\zeta_{i}-\eta_{i}=0$ for all $i$. Hence $\zeta=\eta$ when $n$ is even.

Now if instead $n=2 m+1$, then (3.11) and (3.12) show that $\mu_{1}=-m \mu_{2}$. The equations in (3.6) and (3.7) imply

$$
r^{-m \mu_{2}} s^{(m+1) \mu_{2}}=1 \quad \text { and } \quad r^{(m+1) \mu_{2}} s^{-m \mu_{2}}=1,
$$

and hence that $(r s)^{\mu_{2}}=1$. Then from (3.13) we see that $s^{(2 m+1) \mu_{2}}=1=r^{(2 m+1) \mu_{2}}$. As not both $r$ and $s$ are roots of unity, $\mu_{2}=0$. From this, the desired conclusion $\zeta=\eta$ follows.

Corollary 3.14. Let $M$ be a finite-dimensional module for $U_{r, s}\left(\mathfrak{s l}_{n}\right)$ or for $U_{r, s}\left(\mathfrak{g l}_{n}\right)$. If $\mathrm{rs}^{-1}$ is not a root of unity, then the elements $e_{i}, f_{i}(1 \leq i<n)$ act nilpotently on $M$.

Proof. Because $M$ is a direct sum of its weight spaces, it suffices to argue that $e_{i}$ and $f_{i}$ act nilpotently on each $M_{\chi}$. As the weights $\widehat{k \alpha_{i}}$ for $k=1,2, \ldots$ are distinct by Proposition 3.5, and $e_{i}^{k} M_{\chi} \subseteq M_{\chi \cdot\left(\widehat{k \alpha_{i}}\right)}$, it must be that some power of $e_{i}$ maps $M_{\chi}$ to 0. A similar argument applies to show that $f_{i}$ is nilpotent also. 


\section{$\S 4 . \quad R$-MATRIX AND QUANTUM CASImir OPERATOR}

Let $\mathcal{O}$ denote the category of modules $M$ for $\widetilde{U}=U_{r, s}\left(\mathfrak{g l}_{n}\right)$ which satisfy the conditions:

$(\mathcal{O} 1) \widetilde{U}^{0}$ acts semisimply on $M$, and the set $\operatorname{wt}(M)$ of weights of $M$ belongs to $\Lambda$ : $M=\bigoplus_{\lambda \in \operatorname{wt}(M)} M_{\lambda}$, where $M_{\lambda}=\left\{m \in M \mid a_{i} \cdot m=r^{\left\langle\epsilon_{i}, \lambda\right\rangle}, \quad b_{i} \cdot m=s^{\left\langle\epsilon_{i}, \lambda\right\rangle}\right.$ for all $i\}$;

$(\mathcal{O} 2) \operatorname{dim}_{\mathbb{K}} M_{\lambda}<\infty$ for all $\lambda \in \operatorname{wt}(M)$;

(O3) wt $(M) \subseteq \bigcup_{\mu \in F}\left(\mu-Q^{+}\right)$for some finite set $F \subset \Lambda$.

The morphisms in $\mathcal{O}$ are $\widetilde{U}$-module homomorphisms.

Category $\mathcal{O}$ is closed under tensor product. For any two modules $M$ and $M^{\prime}$ in $\mathcal{O}$, we construct a $\widetilde{U}$-module isomorphism $R_{M^{\prime}, M}: M^{\prime} \otimes M \rightarrow M \otimes M^{\prime}$, by the method used by Jantzen [Ja, Chap. 7] for the quantum groups $U_{q}(\mathfrak{g})$. These isomorphisms work equally well for $U$-modules.

The map $R_{M^{\prime}, M}$ is the composite of three linear transformations $P, \widetilde{f}, \Theta$, which we now describe:

(i) $P=P_{M^{\prime}, M}: M^{\prime} \otimes M \rightarrow M \otimes M^{\prime}, \quad P\left(m^{\prime} \otimes m\right)=m \otimes m^{\prime}$.

(ii) $\tilde{f}=\widetilde{f}_{M, M^{\prime}}: M \otimes M^{\prime} \rightarrow M^{\prime} \otimes M$ is such that $\widetilde{f}\left(m \otimes m^{\prime}\right)=f(\lambda, \mu)\left(m \otimes m^{\prime}\right)$ when $m \in M_{\lambda}$ and $m^{\prime} \in M_{\mu}^{\prime}$, where the map $f: \Lambda \times \Lambda \rightarrow \mathbb{K}^{\#}$ is defined as follows.

Suppose that $\alpha_{n}=\epsilon_{n}$ so that $\alpha_{i}+\alpha_{i+1}+\cdots+\alpha_{n}=\epsilon_{i}$ for $i=1, \ldots, n$. Let $\Lambda=\mathbb{Z} \alpha_{1} \oplus \cdots \oplus \mathbb{Z} \alpha_{n}=\mathbb{Z} \epsilon_{1} \oplus \cdots \oplus \mathbb{Z} \epsilon_{n}$, (the weight lattice of $\mathfrak{g l}_{n}$ ). If $\lambda=\sum_{i=1}^{n} \lambda_{i} \alpha_{i}$ is in the weight lattice $\Lambda$, we define

$$
\begin{aligned}
& \omega_{\lambda}=\omega_{1}^{\lambda_{1}} \cdots \omega_{n-1}^{\lambda_{n-1}} a_{n}^{\lambda_{n}} \\
& \omega_{\lambda}^{\prime}=\left(\omega_{1}^{\prime}\right)^{\lambda_{1}} \cdots\left(\omega_{n-1}^{\prime}\right)^{\lambda_{n-1}} b_{n}^{\lambda_{n}},
\end{aligned}
$$

which agrees with (1.2) in case $\lambda \in Q$. If also $\mu=\sum_{i=1}^{n} \mu_{i} \alpha_{i}$ is in $\Lambda$, we define

$$
f(\lambda, \mu)=\left(\omega_{\mu}^{\prime}, \omega_{\lambda}\right)^{-1} .
$$

The values of this bilinear form are given by (2.1) and (2.3). It may be checked that for all $\lambda, \mu, \nu \in \Lambda$ and $1 \leq i, j<n$, the following hold:

$$
\begin{aligned}
f(\lambda+\mu, \nu) & =f(\lambda, \nu) f(\mu, \nu) \\
f(\lambda, \mu+\nu) & =f(\lambda, \mu) f(\lambda, \nu) \\
f\left(\alpha_{j}, \mu\right) & =r^{-\left\langle\epsilon_{j}, \mu\right\rangle} s^{-\left\langle\epsilon_{j+1}, \mu\right\rangle} \\
f\left(\lambda, \alpha_{i}\right) & =r^{\left\langle\epsilon_{i+1}, \lambda\right\rangle} s^{\left\langle\epsilon_{i}, \lambda\right\rangle} .
\end{aligned}
$$

We will need to compute

$$
f\left(\epsilon_{i}, \epsilon_{j}\right)=f\left(\alpha_{i}+\cdots+\alpha_{n}, \alpha_{j}+\cdots+\alpha_{n}\right) .
$$


Supposing first that $1 \leq i, j<n$, by (4.3) we have

$$
\begin{aligned}
f\left(\epsilon_{i}, \epsilon_{j}\right) & =\left(\omega_{\alpha_{j}+\cdots+\alpha_{n}}^{\prime}, a_{n}\right)^{-1} \prod_{k=i}^{n-1} f\left(\alpha_{k}, \alpha_{j}+\cdots+\alpha_{n}\right) \\
& =r \prod_{k=i}^{n-1} r^{-\left\langle\epsilon_{k}, \alpha_{j}+\cdots+\alpha_{n}\right\rangle} s^{-\left\langle\epsilon_{k+1}, \alpha_{j}+\cdots+\alpha_{n}\right\rangle} \\
& =r \prod_{k=i}^{n-1} r^{-\left\langle\epsilon_{k}, \epsilon_{j}\right\rangle} s^{-\left\langle\epsilon_{k+1}, \epsilon_{j}\right\rangle} \\
& = \begin{cases}s^{-1} & \text { if } i<j \\
r & \text { if } i=j \\
r & \text { if } i>j .\end{cases}
\end{aligned}
$$

Now if $1 \leq i<n$, then

$$
\begin{aligned}
f\left(\epsilon_{i}, \epsilon_{n}\right) & =f\left(\alpha_{i}+\cdots+\alpha_{n}, \alpha_{n}\right)=\left(b_{n}, \omega_{i}\right)^{-1} \cdots\left(b_{n}, \omega_{n-1}\right)^{-1}\left(b_{n}, a_{n}\right)^{-1}=s^{-1} \\
f\left(\epsilon_{n}, \epsilon_{i}\right) & =f\left(\alpha_{n}, \alpha_{i}+\cdots+\alpha_{n}\right)=\left(\omega_{i}, a_{n}\right)^{-1} \cdots\left(\omega_{n-1}, a_{n}\right)^{-1}\left(b_{n}, a_{n}\right)^{-1}=r \quad \text { and } \\
f\left(\epsilon_{n}, \epsilon_{n}\right) & =\left(b_{n}, a_{n}\right)=1 .
\end{aligned}
$$

As a result, the following holds:

Lemma 4.4. For all $1 \leq i, j \leq n$, we have

$$
f\left(\epsilon_{i}, \epsilon_{j}\right)= \begin{cases}s^{-1} & \text { if } i<j \\ 1 & \text { if } i=j \\ r & \text { if } i>j .\end{cases}
$$

(iii) Now we turn our attention to the construction of our final mapping. Observe it is a consequence of (R2) and (R3) that the subalgebra $U^{+}$of $\widetilde{U}$ ( or of $U=U_{r, s}\left(\mathfrak{s l}_{n}\right)$ ) generated by 1 and $e_{i}(1 \leq i<n)$ has the following decomposition

$$
U^{+}=\bigoplus_{\zeta \in Q^{+}} U_{\zeta}^{+}
$$

where

$$
U_{\zeta}^{+}=\left\{z \in U^{+} \mid a_{i} z=r^{\left\langle\epsilon_{i}, \zeta\right\rangle} z a_{i}, \quad b_{i} z=s^{\left\langle\epsilon_{i}, \zeta\right\rangle} z b_{i}, \quad(1 \leq i<n)\right\}
$$

The weight space $U_{\zeta}^{+}$is spanned by all the monomials $e_{i_{1}} \cdots e_{i_{\ell}}$ such that $\alpha_{i_{1}}+$ $\cdots+\alpha_{i_{\ell}}=\zeta$.

Similarly, the subalgebra $U^{-}$generated by 1 and the $f_{i}$ 's has a decomposition $U^{-}=\bigoplus_{\zeta \in Q^{+}} U_{-\zeta}^{-}$, and the spaces $U_{\zeta}^{+}$and $U_{-\zeta}^{-}$are nondegenerately paired.

Since $\Delta\left(e_{i}\right)=e_{i} \otimes 1+\omega_{i} \otimes e_{i}$, we have 


$$
\Delta(x) \in \sum_{0 \leq \nu \leq \zeta} U_{\zeta-\nu}^{+} \omega_{\nu} \otimes U_{\nu}^{+}
$$

for all $x \in U_{\zeta}^{+}$. (In writing this, we are using the standard partial order on $Q$ in which $\nu \leq \zeta$ if $\zeta-\nu \in Q^{+}$.) For each $i$, there are elements $p_{i}(x)$ and $p_{i}^{\prime}(x) \in U_{\zeta-\alpha_{i}}^{+}$ such that

$$
\begin{array}{r}
\Delta(x)=x \otimes 1+\sum_{i=1}^{n-1} p_{i}(x) \omega_{i} \otimes e_{i}+\text { the rest } \\
\Delta(x)=\omega_{\zeta} \otimes x+\sum_{i=1}^{n-1} e_{i} \omega_{\zeta-\alpha_{i}} \otimes p_{i}^{\prime}(x)+\text { the rest }
\end{array}
$$

where in each case "the rest" refers to terms involving products of more than one $e_{j}$ in the second factor (respectively, in the first factor). (Compare the expressions in Lemma 5.2 below.)

Lemma 4.6. (Compare [Ja, Lemma 6.14, 6.17].) For all $x \in U_{\zeta}^{+}, x^{\prime} \in U_{\zeta^{\prime}}^{+}$, and $y \in U^{-}$, the following hold:

(i) $p_{i}\left(x x^{\prime}\right)=r^{\left\langle\epsilon_{i}, \zeta^{\prime}\right\rangle} s^{\left\langle\epsilon_{i+1}, \zeta^{\prime}\right\rangle} p_{i}(x) x^{\prime}+x p_{i}\left(x^{\prime}\right)$.

(ii) $p_{i}^{\prime}\left(x x^{\prime}\right)=p_{i}^{\prime}(x) x^{\prime}+r^{-\left\langle\epsilon_{i+1}, \zeta\right\rangle} s^{-\left\langle\epsilon_{i}, \zeta\right\rangle} x p_{i}^{\prime}(x)$.

(iii) $\left(f_{i} y, x\right)=\left(f_{i}, e_{i}\right)\left(y, p_{i}^{\prime}(x)\right)=(s-r)^{-1}\left(y, p_{i}^{\prime}(x)\right)$.

(iv) $\left(y f_{i}, x\right)=\left(f_{i}, e_{i}\right)\left(y, p_{i}(x)\right)=(s-r)^{-1}\left(y, p_{i}(x)\right)$.

(v) $f_{i} x-x f_{i}=(s-r)^{-1}\left(p_{i}(x) \omega_{i}-\omega_{i}^{\prime} p_{i}^{\prime}(x)\right)$.

Proof. The proofs of (i) and (ii) amount to equating the expressions for $\Delta\left(x x^{\prime}\right)=$ $\Delta(x) \Delta\left(x^{\prime}\right)$. We demonstrate the second:

$$
\begin{aligned}
& \Delta\left(x x^{\prime}\right)= \omega_{\zeta+\zeta^{\prime}} \otimes x x^{\prime}+\sum_{i=1}^{n-1} e_{i} \omega_{\zeta+\zeta^{\prime}-\alpha_{i}} \otimes p_{i}^{\prime}\left(x x^{\prime}\right)+\text { the rest } \\
& \Delta(x) \Delta\left(x^{\prime}\right)=\left(\omega_{\zeta} \otimes x+\sum_{i=1}^{n-1} e_{i} \omega_{\zeta-\alpha_{i}} \otimes p_{i}^{\prime}(x)+\text { the rest }\right) \times \\
& \quad\left(\omega_{\zeta^{\prime}} \otimes x^{\prime}+\sum_{i=1}^{n-1} e_{i} \omega_{\zeta^{\prime}-\alpha_{i}} \otimes p_{i}^{\prime}\left(x^{\prime}\right)+\text { the rest }\right) \\
&=\omega_{\zeta+\zeta^{\prime}} \otimes x x^{\prime} \\
& \quad+\sum_{i=1}^{n-1}\left(e_{i} \omega_{\zeta+\zeta^{\prime}-\alpha_{i}} \otimes p_{i}^{\prime}(x) x^{\prime}+\omega_{\zeta} e_{i} \omega_{\zeta^{\prime}-\alpha_{i}} \otimes x p_{i}^{\prime}\left(x^{\prime}\right)\right)+\text { the rest } \\
&=\omega_{\zeta+\zeta^{\prime}} \otimes x x^{\prime} \\
& \quad+\sum_{i=1}^{n-1} e_{i} \omega_{\zeta+\zeta^{\prime}-\alpha_{i}} \otimes\left(p_{i}^{\prime}(x) x^{\prime}+r^{-\left\langle\epsilon_{i+1}, \zeta\right\rangle} s^{-\left\langle\epsilon_{i}, \zeta\right\rangle} x p_{i}^{\prime}\left(x^{\prime}\right)\right)+\text { the rest },
\end{aligned}
$$


(using Lemma 1.3). Equating terms gives (ii).

Now for (iii) we argue as follows using the second equation of (4.5):

$$
\begin{aligned}
\left(f_{i} y, x\right) & =\sum\left(f_{i}, x_{(1)}\right)\left(y, x_{(2)}\right) \\
& =\left(f_{i}, e_{i} \omega_{\zeta-\alpha_{i}}\right)\left(y, p_{i}^{\prime}(x)\right) \\
& =\left(1 \otimes f_{i}+f_{i} \otimes \omega_{i}^{\prime}, \omega_{\zeta-\alpha_{i}} \otimes e_{i}\right)\left(y, p_{i}^{\prime}(x)\right) \\
& =\left(f_{i}, e_{i}\right)\left(y, p_{i}^{\prime}(x)\right) .
\end{aligned}
$$

Let's begin the proof of $(\mathrm{v})$ by observing that it is true if $x=1 \in U_{0}^{+}$since $p_{i}(1)=p_{i}^{\prime}(1)=0$ for all $i$. The relation in (v) also holds for $x=e_{j}$, because $\Delta\left(e_{j}\right)=e_{j} \otimes 1+w_{j} \otimes e_{j}$ implies that $p_{i}\left(e_{j}\right)=\delta_{i, j}=p_{i}^{\prime}\left(e_{j}\right)$. We suppose the result is true for $x \in U_{\zeta}^{+}$and $x^{\prime} \in U_{\zeta^{\prime}}^{+}$and prove it for $x x^{\prime} \in U_{\zeta+\zeta^{\prime}}^{+}$. Now

$$
\begin{aligned}
& f_{i} x x^{\prime}-x x^{\prime} f_{i}=\left(f_{i} x-x f_{i}\right) x^{\prime}-x\left(f_{i} x^{\prime}-x^{\prime} f_{i}\right) \\
&= \frac{1}{s-r}\left(\left(p_{i}(x) \omega_{i}-\omega_{i}^{\prime} p_{i}^{\prime}(x)\right) x^{\prime}+x\left(p_{i}\left(x^{\prime}\right) \omega_{i}-\omega_{i}^{\prime} p_{i}^{\prime}\left(x^{\prime}\right)\right)\right) \\
&= \frac{1}{s-r}\left(\left(r^{\left\langle\epsilon_{i}, \zeta^{\prime}\right\rangle} s^{\left\langle\epsilon_{i+1}, \zeta^{\prime}\right\rangle} p_{i}(x) x^{\prime}+x\left(p_{i}\left(x^{\prime}\right)\right) \omega_{i}\right.\right. \\
&\left.\quad-\omega_{i}^{\prime}\left(p_{i}^{\prime}(x) x^{\prime}+r^{-\left\langle\epsilon_{i+1}, \zeta\right\rangle} s^{-\left\langle\epsilon_{i}, \zeta\right\rangle} x p_{i}^{\prime}\left(x^{\prime}\right)\right)\right) \\
&=\frac{1}{s-r}\left(p_{i}\left(x x^{\prime}\right) \omega_{i}-\omega_{i}^{\prime} p\left(x x^{\prime}\right)\right) . \quad \square
\end{aligned}
$$

Assuming for $y \in U_{-\zeta}^{-}$that $p_{i}(y)$ and $p_{i}^{\prime}(y)$ are defined by

$$
\begin{aligned}
& \Delta(y)=y \otimes \omega_{\zeta}^{\prime}+\sum_{i=1}^{n} p_{i}(y) \otimes f_{i} \omega_{\zeta-\alpha_{i}}^{\prime}+\text { the rest } \\
& \Delta(y)=1 \otimes y+\sum_{i=1}^{n} f_{i} \otimes p_{i}^{\prime}(y) \omega_{i}^{\prime}+\text { the rest }
\end{aligned}
$$

the same type of argument produces this analogue of Lemma 4.6:

Lemma 4.8. For all $y \in U_{-\zeta}^{-}, y^{\prime} \in U_{-\zeta^{\prime}}^{-}$, and $x \in U^{+}$, the following hold:

(i) $p_{i}\left(y y^{\prime}\right)=p_{i}(y) y^{\prime}+r^{\left\langle\epsilon_{i}, \zeta^{\prime}\right\rangle} s^{\left\langle\epsilon_{i+1}, \zeta^{\prime}\right\rangle} y p_{i}\left(y^{\prime}\right)$.

(ii) $p_{i}^{\prime}\left(y y^{\prime}\right)=r^{-\left\langle\epsilon_{i+1}, \zeta^{\prime}\right\rangle} s^{-\left\langle\epsilon_{i}, \zeta^{\prime}\right\rangle} p_{i}^{\prime}(y) y^{\prime}+y p_{i}^{\prime}(y)$.

(iii) $\left(y, e_{i} x\right)=\left(f_{i}, e_{i}\right)\left(p_{i}(y), x\right)=(s-r)^{-1}\left(p_{i}(y),(x)\right)$.

(iv) $\left(y, x e_{i}\right)=\left(f_{i}, e_{i}\right)\left(p_{i}^{\prime}(y), x\right)=(s-r)^{-1}\left(p_{i}^{\prime}(y), x\right)$.

(v) $e_{i} y-y e_{i}=(r-s)^{-1}\left(w_{i} p_{i}(y)-p_{i}^{\prime}(y) \omega_{i}^{\prime}\right)$.

Because the spaces $U_{\zeta}^{+}$and $U_{-\zeta}^{-}$are nondegenerately paired, we may select a basis $\left\{u_{k}^{\zeta}\right\}_{k=1}^{d_{\zeta}},\left(d_{\zeta}=\operatorname{dim}_{\mathbb{K}} U_{\zeta}^{+}\right)$, for $U_{\zeta}^{+}$and a dual basis $\left\{v_{k}^{\zeta}\right\}_{k=1}^{d_{\zeta}}$ for $U_{-\zeta}^{-}$. Then for each $x \in U_{\zeta}^{+}$and $y \in U_{-\zeta}^{-}$we have 


$$
x=\sum_{k=1}^{d_{\zeta}}\left(v_{k}^{\zeta}, x\right) u_{k}^{\zeta} \quad \text { and } \quad y=\sum_{k=1}^{d_{\zeta}}\left(y, u_{k}^{\zeta}\right) v_{k}^{\zeta}
$$

For $\zeta \in Q^{+}=\bigoplus_{i=1}^{n-1} \mathbb{Z}_{\geq 0} \alpha_{i}$, we define

$$
\Theta_{\zeta}=\sum_{k=1}^{d_{\zeta}} v_{k}^{\zeta} \otimes u_{k}^{\zeta}
$$

Set $\Theta_{\zeta}=0$ if $\zeta \notin Q^{+}$.

Lemma 4.10. (i) $\left(a_{i} \otimes a_{i}\right) \Theta_{\zeta}=\Theta_{\zeta}\left(a_{i} \otimes a_{i}\right)$;

(ii) $\left(b_{i} \otimes b_{i}\right) \Theta_{\zeta}=\Theta_{\zeta}\left(b_{i} \otimes b_{i}\right)$;

(iii) $\left(e_{i} \otimes 1\right) \Theta_{\zeta}+\left(\omega_{i} \otimes e_{i}\right) \Theta_{\zeta-\alpha_{i}}=\Theta_{\zeta}\left(e_{i} \otimes 1\right)+\Theta_{\zeta-\alpha_{i}}\left(\omega_{i}^{\prime} \otimes e_{i}\right)$;

(iv) $\left(1 \otimes f_{i}\right) \Theta_{\zeta}+\left(f_{i} \otimes \omega_{i}^{\prime}\right) \Theta_{\zeta-\alpha_{i}}=\Theta_{\zeta}\left(1 \otimes f_{i}\right)+\Theta_{\zeta-\alpha_{i}}\left(f_{i} \otimes \omega_{i}\right)$;

for $1 \leq i<n$.

Proof. The first two are easy to check. We demonstrate (iv) and leave (iii) as an exercise. The calculation below will use (iii)-(v) of Lemma 4.6 and (4.9).

$$
\begin{aligned}
\left(1 \otimes f_{i}\right) \Theta_{\zeta}-\Theta_{\zeta} & \left.1 \otimes f_{i}\right) \\
= & \sum_{k} v_{k}^{\zeta} \otimes\left(f_{i} u_{k}^{\zeta}-u_{k}^{\zeta} f_{i}\right) \\
= & \frac{1}{s-r} \sum_{k} v_{k}^{\zeta} \otimes\left(p_{i}\left(u_{k}^{\zeta}\right) \omega_{i}-\omega_{i}^{\prime} p_{i}^{\prime}\left(u_{k}^{\zeta}\right)\right) \\
= & \frac{1}{s-r} \sum_{k} v_{k}^{\zeta} \otimes\left(\sum_{j}\left(v_{j}^{\zeta-\alpha_{i}}, p_{i}\left(u_{k}^{\zeta}\right)\right) u_{j}^{\zeta-\alpha_{j}}\right) \omega_{i} \\
& -\frac{1}{s-r} \sum_{k} v_{k}^{\zeta} \otimes \omega_{i}^{\prime}\left(\sum_{j}\left(v_{j}^{\zeta-\alpha_{i}}, p_{i}^{\prime}\left(u_{k}^{\zeta}\right)\right) u_{j}^{\zeta-\alpha_{j}}\right) \\
& \\
& -\sum_{k} v_{k}^{\zeta} \otimes\left(\sum_{j}\left(v_{j}^{\zeta-\alpha_{i}} f_{i}, u_{k}^{\zeta}\right) u_{j}^{\zeta-\alpha_{i}}\right) \omega_{i} \otimes \omega_{i}^{\prime}\left(\sum_{j}\left(f_{i} v_{j}^{\zeta-\alpha_{i}}, u_{k}^{\zeta}\right) u_{j}^{\zeta-\alpha_{j}}\right) \\
= & \sum_{j}\left(\sum_{k}\left(v_{j}^{\zeta-\alpha_{i}} f_{i}, u_{k}^{\zeta}\right) v_{k}^{\zeta}\right) \otimes u_{j}^{\zeta-\alpha_{i}} \omega_{i} \\
& -\sum_{j}\left(\sum_{k}\left(f_{i} v_{j}^{\zeta-\alpha_{i}}, u_{k}^{\zeta}\right) v_{k}^{\zeta}\right) \otimes \omega_{i}^{\prime} u_{j}^{\zeta-\alpha_{j}}
\end{aligned}
$$




$$
\begin{aligned}
& =\sum_{j} v_{j}^{\zeta-\alpha_{i}} f_{i} \otimes u_{j}^{\zeta-\alpha_{i}} \omega_{i}-\sum_{j} f_{i} v_{j}^{\zeta-\alpha_{i}} \otimes \omega_{i}^{\prime} u_{j}^{\zeta-\alpha_{j}} \\
& =\Theta_{\zeta-\alpha_{i}}\left(f_{i} \otimes \omega_{i}\right)-\left(f_{i} \otimes \omega_{i}^{\prime}\right) \Theta_{\zeta-\alpha_{i}} .
\end{aligned}
$$

Consequently, (iv) holds.

We now define

$$
\Theta=\sum_{\zeta \in Q^{+}} \Theta_{\zeta}
$$

(which we can think of as living in the completion of $\widetilde{U} \otimes \widetilde{U}$ where infinite sums are allowed). For fixed $\widetilde{U}$-modules $M$ and $M^{\prime}$ in $\mathcal{O}$, we may apply $\Theta$ to their tensor product:

$$
\Theta=\Theta_{M, M^{\prime}}: M \otimes M^{\prime} \rightarrow M \otimes M^{\prime}
$$

Note that $\Theta_{\zeta}: M_{\lambda} \otimes M_{\mu}^{\prime} \rightarrow M_{\lambda-\zeta} \otimes M_{\mu+\zeta}^{\prime}$ for all $\lambda, \mu \in \Lambda$, and because of condition $(\mathcal{O} 3)$, there are only finitely many $\zeta \in Q^{+}$such that $M_{\mu+\zeta}^{\prime} \neq 0$. Hence, this is a well-defined linear transformation on $M \otimes M^{\prime}$.

We can choose countable bases of weight vectors for both $M$ and $M^{\prime}$ and their tensor products as a basis for $M \otimes M^{\prime}$. Then ordering this basis appropriately shows that each $\Theta_{\zeta}$ with $\zeta>0$ has a strictly upper triangular matrix. Because $\Theta_{0}=1 \otimes 1$ acts as the identity transformation on $M \otimes M^{\prime}, \Theta_{M, M^{\prime}}$ is an invertible transformation.

Theorem 4.11. Let $M$ and $M^{\prime}$ be modules in $\mathcal{O}$. Then the map

$$
\Theta \circ \tilde{f} \circ P: M^{\prime} \otimes M \rightarrow M \otimes M^{\prime}
$$

is an isomorphism of $\widetilde{U}$-modules.

Proof. Since each of the maps is invertible, once we show that $\Theta \circ \widetilde{f} \circ P$ is a $\widetilde{U}$-module homomorphism, we will be done. The proof amounts to verifying that

$$
\Delta(a)(\Theta \circ \tilde{f} \circ P)\left(m^{\prime} \otimes m\right)=(\Theta \circ \tilde{f} \circ P) \Delta(a)\left(m^{\prime} \otimes m\right)
$$

holds for all $a \in \widetilde{U}, m \in M_{\lambda}$ and $m^{\prime} \in M_{\mu}^{\prime}$. Because $\Delta$ is an algebra homomorphism, it suffices to check (4.12) on the generators $e_{i}, f_{i}, a_{i}, b_{i}$. We will present the computation just for $a=e_{i}$. In this case, the right side of (4.12) becomes 


$$
\begin{aligned}
(\Theta \circ \tilde{f} \circ P) \Delta\left(e_{i}\right)\left(m^{\prime} \otimes m\right)= & (\Theta \circ \tilde{f} \circ P)\left(e_{i} m^{\prime} \otimes m+\omega_{i} m^{\prime} \otimes e_{i} m\right) \\
= & (\Theta \circ \tilde{f})\left(m \otimes e_{i} m^{\prime}+e_{i} m \otimes \omega_{i} m^{\prime}\right) \\
= & f\left(\lambda, \mu+\alpha_{i}\right) \Theta\left(m \otimes e_{i} m^{\prime}\right)+f\left(\lambda+\alpha_{i}, \mu\right) \Theta\left(e_{i} m \otimes \omega_{i} m^{\prime}\right) \\
= & f\left(\lambda, \mu+\alpha_{i}\right)\left(\sum_{\zeta} \Theta_{\zeta}\right)\left(1 \otimes e_{i}\right)\left(m \otimes m^{\prime}\right) \\
& \quad+f\left(\lambda+\alpha_{i}, \mu\right)\left(\sum_{\zeta} \Theta_{\zeta}\right)\left(e_{i} \otimes \omega_{i}\right)\left(m \otimes m^{\prime}\right)
\end{aligned}
$$

Now let's compute the left side using (iii) of Lemma 4.10:

$$
\begin{aligned}
& \Delta\left(e_{i}\right)(\Theta \circ \tilde{f} \circ P)\left(m^{\prime} \otimes m\right)= f(\lambda, \mu) \Delta\left(e_{i}\right) \Theta\left(m \otimes m^{\prime}\right) \\
&= f(\lambda, \mu)\left(e_{i} \otimes 1\right)\left(\sum_{\zeta} \Theta_{\zeta}\right)\left(m \otimes m^{\prime}\right) \\
&+f(\lambda, \mu)\left(\omega_{i} \otimes e_{i}\right)\left(\sum_{\zeta} \Theta_{\zeta-\alpha_{i}}\right)\left(m \otimes m^{\prime}\right) \\
&= f(\lambda, \mu)\left(\sum_{\zeta} \Theta_{\zeta}\right)\left(e_{i} m \otimes m^{\prime}\right) \\
&+f(\lambda, \mu)\left(\sum_{\zeta} \Theta_{\zeta-\alpha_{i}}\right)\left(\omega_{i}^{\prime} m \otimes e_{i} m^{\prime}\right) \\
&=f(\lambda, \mu) r^{-\left\langle\epsilon_{i}, \mu\right\rangle} s^{-\left\langle\epsilon_{i+1}, \mu\right\rangle}\left(\sum_{\zeta} \Theta_{\zeta}\right)\left(e_{i} m \otimes \omega_{i} m^{\prime}\right) \\
& \quad+f(\lambda, \mu) r^{\left\langle\epsilon_{i+1}, \lambda\right\rangle} s^{\left\langle\epsilon_{i}, \lambda\right\rangle}\left(\sum_{\zeta} \Theta_{\zeta-\alpha_{i}}\right)\left(m \otimes e_{i} m^{\prime}\right) .
\end{aligned}
$$

This expression can be seen to equal the previous one by (4.3). (Note in this computation we have made liberal use of the fact that $\sum_{\zeta} \Theta_{\zeta}=\sum_{\zeta} \Theta_{\zeta-\alpha_{i}}$ because of our convention that $\Theta_{\eta}=0$ whenever $\eta \notin Q^{+}$.)

\section{Quantum Casimir operator.}

In this subsection, we construct a quantum Casimir operator which commutes with the action of $\widetilde{U}$ on any $\widetilde{U}$-module in $\mathcal{O}$.

Again letting $\left\{u_{k}^{\zeta}\right\}_{k=1}^{d_{\zeta}}$ and $\left\{v_{k}^{\zeta}\right\}_{k=1}^{d_{\zeta}}$ be dual bases for $U_{\zeta}^{+}$and $U_{-\zeta}^{-}$respectively $\left(d_{\zeta}=\operatorname{dim}_{\mathbb{K}} U_{\zeta}^{+}\right)$, define

$$
\Omega=\sum_{\zeta \in Q^{+}} \sum_{k=1}^{d_{\zeta}} S\left(v_{k}^{\zeta}\right) u_{k}^{\zeta},
$$

where $S$ denotes the antipode. Note that by (3.4), $\Omega$ preserves the weight spaces of any $M \in \mathcal{O}$. 
Lemma 4.16. For $M \in \mathcal{O}$, assume $m \in M_{\lambda}$. Then

(i) $\Omega e_{i} . m=\left(r s^{-1}\right)^{-\left\langle\alpha_{i}, \lambda+\alpha_{i}\right\rangle} e_{i} \Omega . m$,

(ii) $\Omega f_{i} \cdot m=\left(r s^{-1}\right)^{\left\langle\alpha_{i}, \lambda\right\rangle} f_{i} \Omega . m$.

Proof. Apply $\mathfrak{m} \circ(S \otimes 1)$ to Lemma 4.10 (iii), where $\mathfrak{m}$ is the multiplication map. As $S$ is an algebra anti-automorphism, this yields

$$
\begin{aligned}
-\sum_{k=1}^{d_{\zeta}} S\left(v_{k}^{\zeta}\right) \omega_{i}^{-1} e_{i} u_{k}^{\zeta} & +\sum_{k=1}^{d_{\zeta-\alpha_{i}}} S\left(v_{k}^{\zeta-\alpha_{i}}\right) \omega_{i}^{-1} e_{i} u_{k}^{\zeta-\alpha_{i}} \\
& =-\sum_{k=1}^{d_{\zeta}} \omega_{i}^{-1} e_{i} S\left(v_{k}^{\zeta}\right) u_{k}^{\zeta}+\sum_{k=1}^{d_{\zeta-\alpha_{i}}}\left(\omega_{i}^{\prime}\right)^{-1} S\left(v_{k}^{\zeta-\alpha_{i}}\right) u_{k}^{\zeta-\alpha_{i}} e_{i}
\end{aligned}
$$

Now act on $m \in M_{\lambda}$ with the result, and sum over all $\zeta \in Q^{+}$. The two sums on the left side cancel, while the right side produces

$$
\omega_{i}^{-1} e_{i} \Omega . m=\left(\omega_{i}^{\prime}\right)^{-1} \Omega e_{i} \cdot m .
$$

By definition, $\Omega$ preserves weight spaces, and so $e_{i} \Omega . m, \Omega e_{i} . m \in M_{\lambda+\alpha_{i}}$. Therefore we have

$$
r^{-\left\langle\epsilon_{i}, \lambda+\alpha_{i}\right\rangle} s^{-\left\langle\epsilon_{i+1}, \lambda+\alpha_{i}\right\rangle} e_{i} \Omega . m=r^{-\left\langle\epsilon_{i+1}, \lambda+\alpha_{i}\right\rangle} s^{-\left\langle\epsilon_{i}, \lambda+\alpha_{i}\right\rangle} \Omega e_{i} . m,
$$

which is equivalent to (i).

The proof of (ii) is virtually identical, and uses Lemma 4.10 (iv).

Now we introduce a certain function $g: \Lambda \rightarrow \mathbb{K}^{\#}$. If $\rho$ denotes the half sum of the positive roots, then $2 \rho=\sum_{j=1}^{n}(n+1-2 j) \epsilon_{j} \in \Lambda$. For $\lambda \in \Lambda$, set

$$
g(\lambda)=\left(r s^{-1}\right)^{\frac{1}{2}\langle\lambda+2 \rho, \lambda\rangle} .
$$

Because $\left\langle\rho, \alpha_{i}\right\rangle=1$ for all $i=1, \ldots, n-1$, it is straightforward to verify that

$$
g\left(\lambda+\alpha_{i}\right)=\left(r s^{-1}\right)^{\left\langle\alpha_{i}, \lambda+\alpha_{i}\right\rangle} g(\lambda)
$$

for all $\lambda \in \Lambda, i \in\{1, \ldots, n-1\}$.

For $M \in \mathcal{O}$, define the linear operator $\Xi: M \rightarrow M$ by

$$
\Xi(m)=g(\lambda) m
$$

for all $m \in M_{\lambda}, \lambda \in \Lambda$. 
Theorem 4.20. The operator $\Omega \Xi: M \rightarrow M$ commutes with the action of $\widetilde{U}$ on any module $M \in \mathcal{O}$.

Proof. As $\Omega \Xi$ preserves the weight spaces of $M$, it commutes with the action of $a_{i}, b_{i}(1 \leq i \leq n)$. It remains to show that $\Omega \Xi$ commutes with $e_{i}, f_{i}(1 \leq i<n)$. Let $m \in M_{\lambda}$. By Lemma 4.16 (i) and (4.18), we have

$$
\begin{aligned}
\Omega \Xi\left(e_{i} . m\right) & =g\left(\lambda+\alpha_{i}\right) \Omega e_{i} \cdot m \\
& =\left(r s^{-1}\right)^{\left\langle\alpha_{i}, \lambda+\alpha_{i}\right\rangle} g(\lambda) \Omega e_{i} \cdot m \\
& =g(\lambda) e_{i} \Omega \cdot m=e_{i} \Omega \Xi(m) .
\end{aligned}
$$

The calculation for $f_{i}$ is similar.

\section{$\S 5$. YANG-BAXter EQUATION AND HEXAGON IDENTITIES}

For pairs $M, M^{\prime}$ of $\widetilde{U}$-modules in category $\mathcal{O}$, we will show first that the maps $R_{M, M^{\prime}}=\Theta \circ \widetilde{f} \circ P: M \otimes M^{\prime} \rightarrow M^{\prime} \otimes M$ satisfy the quantum Yang-Baxter equation. That is, given three $\widetilde{U}$-modules $M, M^{\prime}, M^{\prime \prime}$ in $\mathcal{O}$, we have $R_{12} \circ R_{23} \circ R_{12}=$ $R_{23} \circ R_{12} \circ R_{23}$ as maps from $M \otimes M^{\prime} \otimes M^{\prime \prime}$ to $M^{\prime \prime} \otimes M^{\prime} \otimes M$. This abbreviated notation is standard, for example $R_{12}$ is an application of $R_{M, M^{\prime}}$ to the first two of three factors and the identity map on the third factor.

We will need the following inner product relation. If $x \in U_{\gamma}^{+}, y \in U_{-\gamma}^{-}$, and $\zeta, \eta \in Q$, then

$$
\left(y \omega_{\zeta}^{\prime}, x \omega_{\eta}\right)=(y, x)\left(\omega_{\zeta}^{\prime}, \omega_{\eta}\right)
$$

To derive this, we apply (4.5) and (4.7), keeping in mind that we need to take the opposite coproduct in the first position (equivalently, reverse the order of the factors in the second position):

$$
\begin{aligned}
\left(y \omega_{\zeta}^{\prime}, x \omega_{\eta}\right) & =\left(y \otimes \omega_{\zeta}^{\prime}, \Delta(x) \Delta\left(\omega_{\eta}\right)\right) \\
& =\left(y, x \omega_{\eta}\right)\left(\omega_{\zeta}^{\prime}, \omega_{\eta}\right) \\
& =\left(\Delta(y), \omega_{\eta} \otimes x\right)\left(\omega_{\zeta}^{\prime}, \omega_{\eta}\right) \\
& =(y, x)\left(\omega_{\zeta}^{\prime}, \omega_{\eta}\right) .
\end{aligned}
$$

Lemma 5.2. Let $x \in U_{\gamma}^{+}$and $y \in U_{-\gamma}^{-}$. Then

(i) $\Delta(x)=\sum_{0 \leq \zeta \leq \gamma} \sum_{i, j}\left(v_{i}^{\gamma-\zeta} v_{j}^{\zeta}, x\right) u_{i}^{\gamma-\zeta} \omega_{\zeta} \otimes u_{j}^{\zeta}$,

(ii) $\Delta(y)=\sum_{0 \leq \zeta \leq \gamma} \sum_{i, j}\left(y, u_{i}^{\gamma-\zeta} u_{j}^{\zeta}\right) v_{j}^{\zeta} \otimes v_{i}^{\gamma-\zeta} \omega_{\zeta}^{\prime}$.

Proof. As $x \in U_{\gamma}^{+}$, we have $\Delta(x) \in \sum_{0 \leq \zeta \leq \gamma} U_{\gamma-\zeta}^{+} \omega_{\zeta} \otimes U_{\zeta}^{+}$. Let $c_{i j}^{\zeta} \in \mathbb{K}$ be such that

$$
\Delta(x)=\sum_{\zeta, i, j} c_{i j}^{\zeta} u_{i}^{\gamma-\zeta} \omega_{\zeta} \otimes u_{j}^{\zeta} .
$$


Then for all $k, \ell$, and $\nu$, we see from (5.1) that

$$
\begin{aligned}
\left(v_{k}^{\gamma-\nu} v_{\ell}^{\nu}, x\right) & =\left(v_{k}^{\gamma-\nu} \otimes v_{\ell}^{\nu}, \Delta(x)\right) \\
& =\sum_{\zeta, i, j} c_{i, j}^{\zeta}\left(v_{k}^{\gamma-\nu}, u_{i}^{\gamma-\zeta} \omega_{\zeta}\right)\left(v_{\ell}^{\nu}, u_{j}^{\zeta}\right)=c_{k \ell}^{\nu}
\end{aligned}
$$

which proves (i). The argument for (ii) is similar.

Letting $\Theta^{\mathrm{op}}=\sum_{\gamma \in Q^{+}} \sum_{i} u_{i}^{\gamma} \otimes v_{i}^{\gamma}, \Theta_{12}=\sum_{\gamma \in Q^{+}} \sum_{i} v_{i}^{\gamma} \otimes u_{i}^{\gamma} \otimes 1, \Theta_{i j}^{f}=\Theta_{i j} \circ \widetilde{f}_{i j}$, and defining the other expressions in a like manner, we have the following identities for operators on $M \otimes M^{\prime} \otimes M^{\prime \prime}$.

Lemma 5.3. (i) $(\Delta \otimes 1)\left(\Theta^{\mathrm{op}}\right) \circ \widetilde{f}_{31} \circ \widetilde{f}_{32}=\Theta_{31}^{f} \circ \Theta_{32}^{f}$.

(ii) $\widetilde{f}_{31} \circ \widetilde{f}_{32} \circ \Theta_{12}=\Theta_{12} \circ \widetilde{f}_{31} \circ \widetilde{f}_{32}$.

Proof. Let $m \in M_{\lambda}, m^{\prime} \in M_{\mu}^{\prime}$, and $m^{\prime \prime} \in M_{\nu}^{\prime \prime}$. Then by Lemma 5.2(i) and (4.9), the left side of (i) applied to $m \otimes m^{\prime} \otimes m^{\prime \prime}$ is

$$
\begin{aligned}
(\Delta \otimes 1)\left(\Theta^{\mathrm{op}}\right) \circ \widetilde{f}_{31} \circ \widetilde{f}_{32} & =f(\nu, \mu) f(\nu, \lambda)(\Delta \otimes 1)\left(\sum_{\gamma, k} u_{k}^{\gamma} \otimes v_{k}^{\gamma}\right) \\
& =f(\nu, \mu) f(\nu, \lambda) \sum_{\gamma, k} \sum_{\zeta, i, j}\left(v_{i}^{\gamma-\zeta} v_{j}^{\zeta}, u_{k}^{\gamma}\right) u_{i}^{\gamma-\zeta} \omega_{\zeta} \otimes u_{j}^{\zeta} \otimes v_{k}^{\gamma} \\
& =f(\nu, \mu) f(\nu, \lambda) \sum_{\gamma, \zeta, i, j} u_{i}^{\gamma-\zeta} \omega_{\zeta} \otimes u_{j}^{\zeta} \otimes\left(\sum_{k}\left(v_{i}^{\gamma-\zeta} v_{j}^{\zeta}, u_{k}^{\gamma}\right) v_{k}^{\gamma}\right) \\
& =f(\nu, \mu) f(\nu, \lambda) \sum_{\gamma, \zeta, i, j} u_{i}^{\gamma-\zeta} \omega_{\zeta} \otimes u_{j}^{\zeta} \otimes v_{i}^{\gamma-\zeta} v_{j}^{\zeta} .
\end{aligned}
$$

On the other hand,

$$
\begin{aligned}
\Theta_{31}^{f} \circ \Theta_{32}^{f}\left(m \otimes m^{\prime} \otimes m^{\prime \prime}\right) & =f(\nu, \mu) \sum_{\eta, \zeta, i, j} f(\nu-\zeta, \lambda) u_{i}^{\eta} m \otimes u_{j}^{\zeta} m^{\prime} \otimes v_{i}^{\eta} v_{j}^{\zeta} m^{\prime \prime} \\
& =f(\nu, \mu) f(\nu, \lambda) \sum_{\eta, \zeta, i, j} f(-\zeta, \lambda) u_{i}^{\eta} \otimes u_{j}^{\zeta} \otimes v_{i}^{\eta} v_{j}^{\zeta}\left(m \otimes m^{\prime} \otimes m^{\prime \prime}\right) .
\end{aligned}
$$

Changing variables in the first expression above to $\eta=\gamma-\zeta$, and noticing that $\omega_{\zeta} \cdot m=f(-\zeta, \lambda) m$, we obtain the second expression, proving (i).

Identity (ii) results from a simple calculation using (4.3).

We are now ready to verify the quantum Yang-Baxter equation. 
Theorem 5.4. (Compare [Ja, §7.6].) $R_{12} \circ R_{23} \circ R_{12}=R_{23} \circ R_{12} \circ R_{23}$ as maps from $M \otimes M^{\prime} \otimes M^{\prime \prime}$ to $M^{\prime \prime} \otimes M^{\prime} \otimes M$.

Proof. Note that $P_{\sigma} \circ \Theta_{i j}^{f}=\Theta_{\sigma(i) \sigma(j)}^{f} \circ P_{\sigma}$ for all permutations $\sigma$, and that the $\tilde{f}_{i j}$ commute with one another. Applying Lemma 5.3 and Theorem 4.11, we have

$$
\begin{aligned}
R_{12} \circ R_{23} \circ R_{12} & =P_{12} \circ P_{23} \circ \Theta_{31}^{f} \circ \Theta_{32}^{f} \circ R_{12} \\
& =P_{12} \circ P_{23} \circ(\Delta \otimes 1)\left(\Theta^{\mathrm{op}}\right) \circ \widetilde{f}_{31} \circ \widetilde{f}_{32} \circ \Theta_{12} \circ \tilde{f}_{12} \circ P_{12} \\
& =P_{12} \circ P_{23} \circ(\Delta \otimes 1)\left(\Theta^{\mathrm{op}}\right) \circ \Theta_{12} \circ \widetilde{f}_{31} \circ \tilde{f}_{32} \circ \tilde{f}_{12} \circ P_{12} \\
& =P_{12} \circ P_{23} \circ(\Delta \otimes 1)\left(\Theta^{\mathrm{op}}\right) \circ \Theta_{12} \circ \tilde{f}_{12} \circ P_{12} \circ \widetilde{f}_{32} \circ \widetilde{f}_{31} \\
& =P_{12} \circ P_{23} \circ(\Delta \otimes 1)\left(\Theta^{\mathrm{op}}\right) \circ R_{12} \circ \widetilde{f}_{32} \circ \widetilde{f}_{31} \\
& =P_{12} \circ P_{23} \circ R_{12} \circ(\Delta \otimes 1)\left(\Theta^{\mathrm{op}}\right) \circ \widetilde{f}_{32} \circ \widetilde{f}_{31} \\
& =P_{12} \circ P_{23} \circ \Theta_{12}^{f} \circ P_{12} \circ \Theta_{31}^{f} \circ \Theta_{32}^{f} \\
& =\Theta_{23}^{f} \circ P_{12} \circ P_{23} \circ P_{12} \circ \Theta_{31}^{f} \circ \Theta_{32}^{f} \\
& =\Theta_{23}^{f} \circ P_{23} \circ P_{12} \circ P_{23} \circ \Theta_{31}^{f} \circ \Theta_{32}^{f} \\
& =\Theta_{23}^{f} \circ P_{23} \circ \Theta_{12}^{f} \circ P_{12} \circ \Theta_{23}^{f} \circ P_{23} \\
& =R_{23} \circ R_{12} \circ R_{23} . \quad \square
\end{aligned}
$$

Next we will verify the hexagon identities. For this we require two additional lemmas regarding operators on $M \otimes M^{\prime} \otimes M^{\prime \prime}$.

Lemma 5.5. $(\Delta \otimes 1)\left(\Theta_{\gamma}\right)=\sum_{0 \leq \zeta \leq \gamma}\left(\Theta_{\gamma-\zeta}\right)_{23}\left(\Theta_{\zeta}\right)_{13}\left(1 \otimes \omega_{\zeta}^{\prime} \otimes 1\right)$, and $(1 \otimes \Delta)\left(\Theta_{\gamma}\right)=\sum_{0 \leq \zeta \leq \gamma}\left(\Theta_{\gamma-\zeta}\right)_{12}\left(\Theta_{\zeta}\right)_{13}\left(1 \otimes \omega_{\zeta} \otimes 1\right)$.

Proof. By the definition of $\Theta_{\gamma}$, Lemma 5.2(ii), and (4.9), we have

$$
\begin{aligned}
(\Delta \otimes 1)\left(\Theta_{\gamma}\right) & =\sum_{k} \Delta\left(v_{k}^{\gamma}\right) \otimes u_{k}^{\gamma} \\
& =\sum_{k} \sum_{\zeta, i, j}\left(v_{k}^{\gamma}, u_{i}^{\gamma-\zeta} u_{j}^{\zeta}\right) v_{j}^{\zeta} \otimes v_{i}^{\gamma-\zeta} \omega_{\zeta}^{\prime} \otimes u_{k}^{\gamma} \\
& =\sum_{\zeta, i, j} v_{j}^{\zeta} \otimes v_{i}^{\gamma-\zeta} \omega_{\zeta}^{\prime} \otimes\left(\sum_{k}\left(v_{k}^{\gamma}, u_{i}^{\gamma-\zeta} u_{j}^{\zeta}\right) u_{k}^{\gamma}\right) \\
& =\sum_{\zeta, i, j} v_{j}^{\zeta} \otimes v_{i}^{\gamma-\zeta} \omega_{\zeta}^{\prime} \otimes u_{i}^{\gamma-\zeta} u_{j}^{\zeta} \\
& =\sum_{0 \leq \zeta \leq \gamma}\left(\Theta_{\gamma-\zeta}\right)_{23}\left(\Theta_{\zeta}\right)_{13}\left(1 \otimes \omega_{\zeta}^{\prime} \otimes 1\right) .
\end{aligned}
$$

The second identity may be checked in just the same way. 
Lemma 5.6. $\tilde{f}_{12} \circ\left(\Theta_{\eta}\right)_{13}=\left(\Theta_{\eta}\right)_{13} \circ\left(1 \otimes \omega_{\eta} \otimes 1\right) \circ \widetilde{f}_{12}$, and $\widetilde{f}_{23} \circ\left(\Theta_{\eta}\right)_{13}=\left(\Theta_{\eta}\right)_{13} \circ\left(1 \otimes \omega_{\eta}^{\prime} \otimes 1\right) \circ \widetilde{f}_{23}$.

Proof. Let $m \in M_{\lambda}, m^{\prime} \in M_{\mu}^{\prime}, m^{\prime \prime} \in M_{\nu}^{\prime \prime}$. Then

$$
\begin{aligned}
\tilde{f}_{12} \circ\left(\Theta_{\eta}\right)_{13}\left(m \otimes m^{\prime} \otimes m^{\prime \prime}\right) & =f(\lambda-\eta, \mu) \sum_{i} v_{i}^{\eta} m \otimes m^{\prime} \otimes u_{i}^{\eta} m^{\prime \prime} \\
& =f(\lambda, \mu) f(-\eta, \mu) \sum_{i} v_{i}^{\eta} m \otimes m^{\prime} \otimes u_{i}^{\eta} m^{\prime \prime} \\
& =f(\lambda, \mu) \sum_{i} v_{i}^{\eta} m \otimes \omega_{\eta} m^{\prime} \otimes u_{i}^{\eta} m^{\prime \prime} \\
& =\left(\Theta_{\eta}\right)_{13} \circ\left(1 \otimes \omega_{\eta} \otimes 1\right) \circ \tilde{f}_{12}\left(m \otimes m^{\prime} \otimes m^{\prime \prime}\right) .
\end{aligned}
$$

The second identity can be shown using $\omega_{\eta}^{\prime} m^{\prime}=f(\mu, \eta) m^{\prime}$.

We continue with our assumption that $M, M^{\prime}$, and $M^{\prime \prime}$ are $\widetilde{U}$-modules in $\mathcal{O}$. To verify the hexagon identities, let $\widetilde{f}^{\prime}$ denote the transformation on $M^{\prime \prime} \otimes M \otimes M^{\prime}$ taking $m^{\prime \prime} \otimes m \otimes m^{\prime} \in M_{\nu}^{\prime \prime} \otimes M_{\lambda} \otimes M_{\mu}^{\prime}$ to $f(\nu, \lambda+\mu) m^{\prime \prime} \otimes m \otimes m^{\prime}$. Let $\tilde{f}^{\prime \prime}$ be the transformation on $M^{\prime} \otimes M^{\prime \prime} \otimes M$ taking $m^{\prime} \otimes m^{\prime \prime} \otimes m \in M_{\mu}^{\prime} \otimes M_{\nu}^{\prime \prime} \otimes M_{\lambda}$ to $f(\mu+\nu, \lambda) m^{\prime} \otimes m^{\prime \prime} \otimes m$. As in [Ja, Thm. 3.18], the hexagon identities are equivalent to $R_{12} \circ R_{23}=(1 \otimes \Delta)(\Theta) \circ \tilde{f}^{\prime} \circ P_{12} \circ P_{23}$ as maps from $M \otimes\left(M^{\prime} \otimes M^{\prime \prime}\right) \rightarrow$ $\left(M^{\prime \prime} \otimes M\right) \otimes M^{\prime}$, and $R_{23} \circ R_{12}=(\Delta \otimes 1)(\Theta) \circ \tilde{f}^{\prime \prime} \circ P_{23} \circ P_{12}$ as maps from $\left(M \otimes M^{\prime}\right) \otimes M^{\prime \prime}$ to $M^{\prime} \otimes\left(M^{\prime \prime} \otimes M\right)$.

Theorem 5.7. The hexagon identities hold, that is,

(i) $R_{12} \circ R_{23}=(1 \otimes \Delta)(\Theta) \circ \tilde{f}^{\prime} \circ P_{12} \circ P_{23}$, and

(ii) $R_{23} \circ R_{12}=(\Delta \otimes 1)(\Theta) \circ \tilde{f}^{\prime \prime} \circ P_{23} \circ P_{12}$.

Proof. Let $m \otimes m^{\prime} \otimes m^{\prime \prime} \in M_{\lambda} \otimes M_{\mu}^{\prime} \otimes M_{\nu}^{\prime \prime}$. By Lemma 5.5, the right side of (i) applied to $m \otimes m^{\prime} \otimes m^{\prime \prime}$ gives

$$
\begin{aligned}
(1 \otimes \Delta)(\Theta) \circ \tilde{f}^{\prime} \circ P_{12} \circ P_{23}\left(m \otimes m^{\prime} \otimes m^{\prime \prime}\right) & \\
& =f(\nu, \lambda+\mu) \sum_{\gamma \in Q^{+}} \sum_{0 \leq \zeta \leq \gamma}\left(\Theta_{\gamma-\zeta}\right)_{12}\left(\Theta_{\zeta}\right)_{13}\left(1 \otimes \omega_{\zeta} \otimes 1\right)\left(m^{\prime \prime} \otimes m \otimes m^{\prime}\right) .
\end{aligned}
$$

On the other hand, by Lemma 5.6, the left side of (i) can be seen to equal

$$
\begin{aligned}
\Theta_{12} \circ \tilde{f}_{12} \circ P_{12} \circ \Theta_{23} \circ \tilde{f}_{23} \circ P_{23}\left(m \otimes m^{\prime} \otimes m^{\prime \prime}\right)=\Theta_{12} \circ \tilde{f}_{12} \circ \Theta_{13} \circ \tilde{f}_{13}\left(m^{\prime \prime} \otimes m \otimes m^{\prime}\right) \\
=\sum_{\eta \in Q^{+}} \Theta_{12} \circ\left(\Theta_{\eta}\right)_{13} \circ\left(1 \otimes \omega_{\eta} \otimes 1\right) \circ \widetilde{f}_{12} \circ \widetilde{f}_{13}\left(m^{\prime \prime} \otimes m \otimes m^{\prime}\right) \\
=f(\nu, \lambda) f(\nu, \mu) \sum_{\eta \in Q^{+}} \Theta_{12} \circ\left(\Theta_{\eta}\right)_{13} \circ\left(1 \otimes \omega_{\eta} \otimes 1\right)\left(m^{\prime \prime} \otimes m \otimes m^{\prime}\right) .
\end{aligned}
$$

Then because $f(\nu, \lambda+\mu)=f(\nu, \lambda) f(\nu, \mu)$, a change of variables shows that this is equal to the right side of (i). The proof of (ii) is similar.

Remark 5.8. $\mathcal{O}$ is a braided monoidal category with braiding $R=R_{M^{\prime}, M}$ for each pair of modules $M^{\prime}, M$ in $\mathcal{O}$. 


\section{§6. ISOMORPHISMS AMONG QUANTUM GROUPS}

We will now investigate isomorphisms among the two-parameter quantum groups, and their connections with multiparameter quantum groups. The case $n=2$ is special, and the two parameters collapse to one in the following sense. Let $r, r^{\prime}, s, s^{\prime} \in \mathbb{K}^{\#}$ and $r \neq s, r^{\prime} \neq s^{\prime}$. If $r s^{-1}=r^{\prime}\left(s^{\prime}\right)^{-1}$, there is an isomorphism of Hopf algebras

$$
\phi: U_{r, s}\left(\mathfrak{s l}_{2}\right) \rightarrow U_{r^{\prime}, s^{\prime}}\left(\mathfrak{s l}_{2}\right)
$$

given by $\phi\left(\omega^{ \pm 1}\right)=\check{\omega}^{ \pm 1}, \phi\left(\left(\omega^{\prime}\right)^{ \pm 1}\right)=\left(\check{\omega}^{\prime}\right)^{ \pm 1}, \phi(e)=\check{e}, \phi(f)=r^{-1} r^{\prime} \check{f}$, where " " " denotes generators of $U_{r^{\prime}, s^{\prime}}\left(\mathfrak{s l}_{2}\right)$. (When dealing with $\mathfrak{s l}_{2}$, we omit the subscript "1" on the generators.) The proof is a simple check that the relations and coproducts are preserved. In particular, if $q$ is a square root of $r s^{-1}$, then $U_{r, s}\left(\mathfrak{s l}_{2}\right) \cong U_{q, q^{-1}}\left(\mathfrak{s l}_{2}\right)$ as Hopf algebras. Therefore the one-parameter quantum group $U_{q}\left(\mathfrak{s l}_{2}\right)$ is isomorphic to the quotient of $U_{r, s}\left(\mathfrak{s l}_{2}\right)$ by the ideal generated by $\omega^{\prime}-\omega^{-1}$.

If $n \geq 3$, there is no such isomorphism, as the following proposition shows.

Proposition 6.1. Let $n \geq 3$, and assume there is an isomorphism of Hopf algebras

$$
\phi: U_{r, s}\left(\mathfrak{s l}_{n}\right) \rightarrow U_{q, q^{-1}}\left(\mathfrak{s l}_{n}\right)
$$

for some $q$. Then $r=q$ and $s=q^{-1}$.

Proof. Let $\phi$ be an isomorphism as hypothesized, and assume

$$
\pi: U_{q, q^{-1}}\left(\mathfrak{s l}_{n}\right) \rightarrow U_{q}\left(\mathfrak{s l}_{n}\right)
$$

is the surjection onto the standard one-parameter quantum group of [Ja] given by $\pi\left(e_{i}\right)=E_{i}, \pi\left(f_{i}\right)=F_{i}, \pi\left(\omega_{i}^{ \pm 1}\right)=K_{i}^{ \pm 1}, \pi\left(\left(\omega_{i}^{\prime}\right)^{ \pm 1}\right)=K_{i}^{\mp 1}$. For $1 \leq i \leq n-1$, we have

$$
\begin{aligned}
\Delta\left(\pi \phi\left(e_{i}\right)\right)=\pi \phi\left(\Delta\left(e_{i}\right)\right) & =\pi \phi\left(e_{i} \otimes 1+\omega_{i} \otimes e_{i}\right) \\
& =\pi \phi\left(e_{i}\right) \otimes 1+\pi \phi\left(\omega_{i}\right) \otimes \pi \phi\left(e_{i}\right)
\end{aligned}
$$

Note that $\pi \phi\left(\omega_{i}\right)$ is necessarily a group-like element. Therefore $\pi \phi\left(e_{i}\right)$ is a skewprimitive element in $U_{q}\left(\mathfrak{s l}_{n}\right)$. By Theorem 5.4.1, Lemma 5.5.5, and the subsequent comments in [M], the set of group-like elements in $U_{q}\left(\mathfrak{s l}_{n}\right)$ is the group $G$ generated by $K_{j}(1 \leq j \leq n-1)$, and the skew-primitive elements together with the group-like elements span the subspace

$$
\sum_{j=1}^{n-1}\left(\mathbb{K} E_{j}+\mathbb{K} F_{j}\right)+\mathbb{K} G
$$

Therefore

$$
\pi \phi\left(e_{i}\right)=\sum_{j=1}^{n-1} \alpha_{j}^{i} E_{j}+\beta_{j}^{i} F_{j}+\sum_{g \in G} \gamma_{g}^{i} g
$$


for some scalars $\alpha_{j}^{i}, \beta_{j}^{i}, \gamma_{g}^{i}(1 \leq i, j \leq n-1, g \in G)$. Consequently

$$
\Delta\left(\pi \phi\left(e_{i}\right)\right)=\sum_{j=1}^{n-1} \alpha_{j}^{i}\left(E_{j} \otimes 1+K_{j} \otimes E_{j}\right)+\beta_{j}^{i}\left(1 \otimes F_{j}+F_{j} \otimes K_{j}^{-1}\right)+\sum_{g \in G} \gamma_{g}^{i} g \otimes g .
$$

By (6.2), this must be equal to

$\sum_{j=1}^{n-1}\left(\alpha_{j}^{i} E_{j} \otimes 1+\beta_{j}^{i} F_{j} \otimes 1+\pi \phi\left(\omega_{i}\right) \otimes \alpha_{j}^{i} E_{j}+\pi \phi\left(\omega_{i}\right) \otimes \beta_{j}^{i} F_{j}\right)+\sum_{g}\left(\gamma_{g}^{i} g \otimes 1+\pi \phi\left(\omega_{i}\right) \otimes \gamma_{g}^{i} g\right)$.

Comparing these two expressions, and noting that $\pi \phi\left(\omega_{i}\right) \neq 1$ as $\phi$ is an isomorphism (or by the comparison of expressions), we see first that

$$
\sum_{j=1}^{n-1} \alpha_{j}^{i} E_{j}+\gamma_{1}^{i} 1=\sum_{j=1}^{n-1}\left(\alpha_{j}^{i} E_{j}+\beta_{j}^{i} F_{j}\right)+\sum_{g \in G} \gamma_{g}^{i} g+\gamma_{1}^{i} \pi \phi\left(\omega_{i}\right)
$$

Therefore $\gamma_{g}^{i}=0$ for all $g$ except $g \in\left\{1, \pi \phi\left(\omega_{i}\right)\right\}, \quad \gamma_{\pi \phi\left(\omega_{i}\right)}^{i}=-\gamma_{1}^{i}$, and all $\beta_{j}^{i}=0$. We now have

$$
\pi \phi\left(e_{i}\right)=\sum_{1 \leq j \leq n-1} \alpha_{j}^{i} E_{j}+\gamma_{1}^{i}\left(1-\pi \phi\left(\omega_{i}\right)\right) .
$$

A further comparison of coproducts yields

$$
\begin{aligned}
\sum_{j=1}^{n-1} \alpha_{j}^{i}\left(E_{j} \otimes 1+K_{j} \otimes E_{j}\right)+\gamma_{1}^{i}\left(1 \otimes 1-\pi \phi\left(\omega_{i}\right) \otimes \pi \phi\left(\omega_{i}\right)\right) \\
=\sum_{j=1}^{n-1} \alpha_{j}^{i}\left(E_{j} \otimes 1+\pi \phi\left(\omega_{i}\right) \otimes E_{j}\right) \\
\quad+\gamma_{1}^{i}\left(1 \otimes 1-\pi \phi\left(\omega_{i}\right) \otimes 1\right)+\gamma_{1}^{i}\left(\pi \phi\left(\omega_{i}\right) \otimes 1-\pi \phi\left(\omega_{i}\right) \otimes \pi \phi\left(\omega_{i}\right)\right),
\end{aligned}
$$

which implies that

$$
\alpha_{j}^{i}\left(K_{j}-\pi \phi\left(\omega_{i}\right)\right)=0, \quad \text { for all } 1 \leq j \leq n-1 .
$$

Thus all $\alpha_{j}^{i}=0$ except possibly one, and some $\alpha_{j_{i}}^{i}$ must be nonzero as $\pi \phi$ is surjective. Therefore $\pi \phi\left(\omega_{i}\right)=K_{j_{i}}$.

Next we will apply $\pi \phi$ to relation (R2') and use the relations in $U_{q}\left(\mathfrak{s l}_{n}\right)$ :

$$
\begin{aligned}
\pi \phi\left(\omega_{i} e_{i}\right) & =\pi \phi\left(r s^{-1} e_{i} \omega_{i}\right) \\
K_{j_{i}}\left(\alpha_{j_{i}}^{i} E_{j_{i}}+\gamma_{1}^{i}\left(1-K_{j_{i}}\right)\right) & =r s^{-1}\left(\alpha_{j_{i}}^{i} E_{j_{i}}+\gamma_{1}^{i}\left(1-K_{j_{i}}\right)\right) K_{j_{i}} \\
\alpha_{j_{i}}^{i} q^{2} E_{j_{i}} K_{j_{i}}+\gamma_{1}^{i} K_{j_{i}}-\gamma_{1}^{i} K_{j_{i}}^{2} & =\alpha_{j_{i}}^{i} r s^{-1} E_{j_{i}} K_{j_{i}}+\gamma_{1}^{i} r s^{-1} K_{j_{i}}-\gamma_{1}^{i} r s^{-1} K_{j_{i}}^{2} .
\end{aligned}
$$

This forces $r s^{-1}=q^{2}$, and $\gamma_{1}^{i}=0$ as $r \neq s$. Applying relation (R2') again, we have

$$
\begin{aligned}
\pi \phi\left(\omega_{i} e_{i+1}\right) & =\pi \phi\left(s e_{i+1} \omega_{i}\right) \\
K_{j_{i}} \alpha_{j_{i+1}}^{i+1} E_{j_{i+1}} & =\alpha_{j_{i+1}}^{i+1} s E_{j_{i+1}} K_{j_{i}} \\
\alpha_{j_{i+1}}^{i+1} q^{\left\langle\alpha_{j_{i}}, \alpha_{j_{i+1}}\right\rangle} E_{j_{i+1}} K_{j_{i}} & =\alpha_{j_{i+1}}^{i+1} s E_{j_{i+1}} K_{j_{i}} .
\end{aligned}
$$

Thus $s=q^{\left\langle\alpha_{j_{i}}, \alpha_{j_{i+1}}\right\rangle}$ must hold. By relations (R5) and (R6), we see that $\left|j_{i}-j_{i+1}\right|=$ 1 , so that in fact $s=q^{-1}$. Combining this with $r s^{-1}=q^{2}$ we now have $r=q$ as well. 


\section{Multiparameter quantum groups.}

Deformations of $G L_{n}$ involving $1+\left(\begin{array}{c}n \\ 2\end{array}\right)$ parameters were constructed independently by several authors (see $[\mathrm{AST}],[\mathrm{R}],[\mathrm{S}]$ ). The dual version is a multiparameter universal enveloping algebra, which was studied by Chin and Musson [ChM] and Dobrev and Parashar [DP]. We will show that our two-parameter quantum groups are essentially special cases of these multiparameter quantum groups, as should be expected. We adopt the notation of Chin and Musson.

The $1+\left(\begin{array}{l}n \\ 2\end{array}\right)$ parameters are denoted $\lambda$ and $p_{i, j}(1 \leq i<j \leq n)$ in $[\mathrm{ChM}]$. Set $\lambda=r s^{-1}$ and $p_{i, j}=s^{-1}$ for all $i<j$. Let $\widehat{U}$ be the Hopf algebra generated by $E_{i}, F_{i}(1 \leq i<n)$ and $K_{i}^{ \pm 1}, L_{i}^{ \pm 1}(1 \leq i \leq n)$ with relations given by

(ChM0) The $K_{i}^{ \pm 1}, L_{j}^{ \pm 1}$ all commute with one another and $K_{i} K_{i}^{-1}=L_{i} L_{i}^{-1}$.

(ChM1) $\quad K_{j} E_{i}=r^{-\delta_{i, j}} s^{-\delta_{i, j-1}} E_{i} K_{j}$ and $K_{j} F_{i}=r^{\delta_{i, j}} s^{\delta_{i, j-1}} F_{i} K_{j}$.

(ChM2) $\quad L_{j} E_{i}=r^{\delta_{i, j-1}} s^{\delta_{i, j}} E_{i} L_{j}$ and $L_{j} F_{i}=r^{-\delta_{i, j-1}} s^{-\delta_{i, j}} F_{i} L_{j}$.

(ChM3) $E_{i} F_{i}-r^{-1} s F_{i} E_{i}=\left(r^{-1} s-1\right)\left(L_{i+1} K_{i+1} L_{i}^{-1} K_{i}^{-1}-1\right)$.

(ChM4) $\quad E_{i} F_{j}=r^{\delta_{i, j+1}} s^{-\delta_{i, j-1}} F_{j} E_{i}$ if $i \neq j$.

(ChM5) $\operatorname{ad}_{\ell}\left(E_{i}\right)^{1-\left\langle\alpha_{i}, \alpha_{j}\right\rangle}\left(E_{j}\right)=0$ and $\operatorname{ad}_{\ell}\left(F_{i}\right)^{1-\left\langle\alpha_{i}, \alpha_{j}\right\rangle}\left(F_{j}\right)=0$ if $i \neq j$.

These relations are given in [ChM, Thm. 4.8] (for more general $\lambda, p_{i, j}$ ) as relations for a Hopf algebra that is defined as a subalgebra of the finite dual $A^{0}$ of a multiparameter quantum function algebra $A$. In addition, Chin and Musson give one more set of conditions: (ChM6) those relations among the $K_{i}, L_{j}$ which determine the structure of the group they generate as a subgroup of the group of units of $A^{0}$. This results in a Hopf algebra $\bar{U}$ (denoted $U$ in their paper). Thus the multiparameter Hopf algebra $\bar{U}$ of Chin and Musson is the quotient of our $\widehat{U}$ by their relations (ChM6) (in case $\lambda=r s^{-1}$ and $p_{i, j}=s^{-1}$ ).

The Hopf structure of $\widehat{U}$ is defined by requiring $K_{i}, L_{i}$ to be group-like elements and

$$
\Delta\left(E_{i}\right)=E_{i} \otimes 1+L_{i+1} L_{i}^{-1} \otimes E_{i}, \quad \Delta\left(F_{i}\right)=F_{i} \otimes 1+K_{i+1} K_{i}^{-1} \otimes F_{i} .
$$

Proposition 6.3. There is a Hopf algebra morphism $\phi: \widehat{U} \rightarrow U_{r, s}\left(\mathfrak{g l}_{n}\right)$ given by

$$
\begin{aligned}
\phi\left(L_{i}\right) & =a_{1} \cdots a_{i-1} b_{i+1}^{-1} \cdots b_{n}^{-1}, \\
\phi\left(K_{i}\right) & =b_{1}^{-1} \cdots b_{i-1}^{-1} a_{i+1} \cdots a_{n}, \\
\phi\left(E_{i}\right) & =-s^{-1}(r-s)^{2} e_{i}, \\
\phi\left(F_{i}\right) & =\left(\omega_{i}^{\prime}\right)^{-1} f_{i} .
\end{aligned}
$$

The proof is just a check that the relations of $\widehat{U}$ are preserved by $\phi$, and that the coproducts of the generators correspond to the coproducts of their images.

Remark 6.4. Note that the kernel and cokernel of $\phi$ are generated by group-like elements. We have $\omega_{i}=\phi\left(L_{i}^{-1} L_{i+1}\right)$ and $\omega_{i}^{\prime}=\phi\left(K_{i} K_{j+1}^{-1}\right)$, which implies that $U_{r, s}\left(\mathfrak{s l}_{n}\right)$ is contained in the image of $\phi$. In case $n=2$, it is easy to see that $\phi$ is an isomorphism. If $n=3$, straightforward calculations show that the cokernel of $\phi$ is precisely $\mathbb{K}\left\langle a_{1}^{ \pm 1}\right\rangle$. 


\section{REFERENCES}

[AST] M. Artin, W. Schelter, and J. Tate, Quantum deformations of $G L_{n}$, Comm. Pure Appl. Math. XLIV (1991), 879-895.

[BL] V.V. Bavula and T.H. Lenagan, Generalized Weyl algebras are tensor Krull minimal, J. Algebra 235 (2001), 315-358.

[B] G. Benkart, Down-up algebras and Witten's deformations of the universal enveloping algebra of $\mathfrak{s l}_{2}$; Recent Progress in Algebra, Contemp. Math., vol. 224, Amer. Math. Soc., 1998, pp. 29-45.

[BR] G. Benkart and T. Roby, Down-up algebras, J. Algebra, 209 (1998), 305-344; Addendum 213 (1999), 378.

[BW1] G. Benkart and S. Witherspoon, A Hopf structure for down-up algebras, Math. Zeitschrift (to appear).

[BW2] G. Benkart and S. Witherspoon, Representations of two-parameter quantum groups and Schur-Weyl duality, preprint.

[CaM] P.A.A.B. Carvalho and I.M. Musson, Down-up algebras and their representation theory, J. Algebra 228 (2000), 286-310.

[ChM] W. Chin and I. M. Musson, Multiparameter quantum enveloping algebras, J. Pure Appl. Algebra 107 (1996), 171-191.

[DP] V. K. Dobrev and P. Parashar, Duality for multiparametric quantum GL(n), J. Phys. A: Math. Gen. 26 (1993), 6991-7002.

[Ja] J.C. Jantzen, Lectures on Quantum Groups, vol. 6, Graduate Studies in Math., Amer. Math. Soc., Providence, 1996.

[Ji] N.H. Jing, Quantum groups with two parameters; Deformation Theory and Quantum Groups with Applications to Mathematical Physics (Amherst, MA, 1990), Contemp. Math., vol. 134, Amer. Math. Soc., 1992, pp. 129-138.

[Jo] A. Joseph, Quantum Groups and Their Primitive Ideals, Ergebnisse der Mathematik und ihrer Grenzgebiete, Springer-Verlag, Berlin, 1995.

[Jor] D.A. Jordan, Down-up algebras and ambiskew polynomial rings, J. Algebra 228 (2000), 311-346.

[KK1] E. Kirkman and J. Kuzmanovich, Primitivity of Noetherian down-up algebras, Comm. Algebra 28 (2000), 2983-2997.

[KK2] E. Kirkman and J. Kuzmanovich, Non-Noetherian down-up algebras, Comm. Algebra 28 (2000), 5255-5268.

[KMP] E.E. Kirkman, I. Musson, and D. Passman, Noetherian down-up algebras, Proc. Amer. Math. Soc. 127 (1999), 3161-3167.

[K] P.P. Kulish, A two-parameter quantum group and gauge transformations (in Russian), Zap. Nauch. Semin. LOMI 180 (1990), 89-93.

$[\mathrm{Ku}] \quad$ R.S. Kulkarni, Down-up algebras and their representations, J. Algebra (to appear).

[M] S. Montgomery, Hopf Algebras and Their Actions on Rings. CBMS Conf. Math. Publ., vol. 82, Amer. Math. Soc., Providence, 1993.

[R] N. Reshetikhin, Multiparameter quantum groups and twisted quasitriangular Hopf algebras, Lett. Math. Phys. 20 (1990), 331-335.

[S] A. Sudbery, Consistent multiparameter quantization of $G L(n)$, J. Phys. A: Math. Gen. (1990), L697-L704. 
[T] M. Takeuchi, A two-parameter quantization of $G L(n)$, Proc. Japan Acad. 66 Ser. A (1990), 112-114.

Department of Mathematics, University of Wisconsin, Madison, Wisconsin 53706 BENKART@MATH.WISC.EDU

Department of Mathematics, University of Massachusetts, Amherst, Massachusetts 01003

(2001-02) Department of Mathematics and Computer Science, Amherst College, Amherst, Massachusetts 01002

WITHER@MATH.UMASS.EDU 